\title{
DISCRIMINATING DISTRIBUTED SENTINEL INVOLVING A NAVIER-STOKES PROBLEM AND PARAMETER IDENTIFICATION*
}

\author{
JEAN VELIN ${ }^{1}$
}

\begin{abstract}
In this paper, we consider a Navier-Stokes system with missing initial data condition and perturbation distributed term or pollution term. We show that a discriminating distributed Sentinel with constraints can be associated to this system and allows to characterize this pollution. Our major result is based on an adapted distributed Carleman Inequality permitting to revisit a study investigated by Lions in [15].

Résumé. Dans ce papier, nous considérons un système de Navier-Stokes pour lequel au terme source est ajouté un terme de pollution et à la donnée initiale est affectée une incertitude ou terme manquant. Nous montrons qu'une Sentinelle distribuée, discriminante avec contraintes peut-être associée à ce système et permet alors de caractériser cette pollution. Notre principale résultat est basé sur une inégalité de Carleman adaptée. Ainsi, nous revoyons une étude abordée par Lions dans [15].
\end{abstract}

\section{Contents}

Introduction

\begin{tabular}{ll}
0.1. & Setting of the problem \\
\hline
\end{tabular}

$\begin{array}{lrr}0.2 . & \text { A null-controllability problem } & 146\end{array}$

$\begin{array}{llr}0.3 . & \text { Related studies } & 148\end{array}$

0.4. Notations and Assumptions $r$

0.5. Main results $r$

1. Proof of Theorem 0.6. Existence of a control 149

1.1. An adapted Observability Inequality 149

1.2. Existence of the best control.

2. Proof of Theorem 0.7. A characterization of $\hat{k}$ via an optimal system 156

2.1. Convergence of the minimizing sequence $\left(k_{\varepsilon}^{n}, q_{\varepsilon}^{n}, \pi_{\varepsilon}^{n}\right) \quad 157$

2.2. Some a priori estimates on the sequence $\left(k_{\varepsilon}, q_{\varepsilon}, \pi_{\varepsilon}\right)$. 159

2.3. A characterization of the sequence control $k_{\varepsilon}$. 160

2.4. New definition of $\hat{k} \quad 164$

3. Proof of Theorem 0.8. A discriminating Sentinel 164

3.1. A use of the concept of sentinel: The identification of the unknown distributed pollution term 165

$\begin{array}{lr}\text { References } & 165\end{array}$

* Laboratoire Analyse Optimisation Contrôle

1 Université des Antilles et de La Guyane, Campus Fouillole 97159 Pointe-à-Pitre GuADELOUPE (FWI).e-mail:jvelin@univ-ag.fr 


\section{INTRODUCTION}

\subsection{Setting of the problem}

Let $N \in\{2 ; 3\}, \Omega$ is a bounded open in $\mathbb{R}^{N}$ with smooth boundary $\Gamma=\partial \Omega$ of class $\mathcal{C}^{2}$. Let $\omega$ be a nonempty bounded open in $\Omega$. $T>0$ is fixed, we denote by $Q=\Omega \times(0, T), \Sigma=\Gamma \times(0, T)$ and $\omega_{T}=\omega \times(0, T)$.

It is well known that the following Navier-Stokes system

$$
\left\{\begin{aligned}
\frac{\partial y}{\partial t}-\Delta y+y \nabla y+\nabla p & =\text { Source term } & & \text { in } Q \\
\text { divy } & =0 & & \text { in } Q \\
y & =0 & & \text { on } \Sigma \\
y(0) & =\text { initial data } & & \text { in } \Omega
\end{aligned}\right.
$$

is a modelling transportation of a flow $y(x, t)$ located in the spatial point $x$ of the domain $\Omega$ and at the time $t \in[0, T]$. Moreover, $y$ is submitted to the pressure $p(x, t)$ and also to different exterior strengths represented by a source term $\xi$.

Generally, the mathematical studies on the existence of solutions for Navier-Stokes equations present a perfect model where the source term and the initial data are well defined (see for instance [18], [21], [22], [3], [4], [7] when $\Omega$ is a bounded domain, ). Therefore, the pollution term denoted by $\lambda \hat{\xi}$ can appears in addition to the natural source term $\xi$. Also, the initial data can be defined with an incertitude $\tau \hat{y}^{0}$. Respectively $\lambda \hat{\xi}$ and $\tau \hat{y}^{0}$ are designed as the pollution term and the missing data or incomplete data.

So, considering the perturbed system

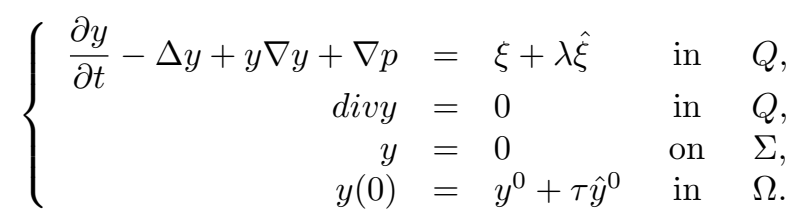

we want to determinate the pollution $\lambda \hat{\xi}$. To do so, we consider an observer $h_{0}$ in an observatory domain assigned $\mathcal{O}$, a non-empty subset in $\Omega$. We denote an observation of $y$ by $y_{o b s}=y \chi_{\mathcal{O}}$ during the time interval $(0, T)$ into the observatory $\mathcal{O}$. Throughout this paper, we suppose that this observation is subject to $M$ different interferences $\left\{\beta_{i} m_{i}\right\}_{i=1, \cdots, M}$. More precisely, if $m_{0} \in\left(L^{2}\left(0, T ; L^{2}(\Omega)\right)\right)^{N}$ is a measurement, we can formulate the observation as follows

$$
y_{\mid o b s}=y \chi_{\mathcal{O}}=m_{0}+\sum_{i=1}^{M} \beta m_{i}
$$

Setting $\mathcal{O}_{T}=\mathcal{O} \times(0, T)$, we can suppose that the functions $m_{1}, m_{2}, \cdots, m_{M}$ belong in $\left(L^{2}\left(\mathcal{O}_{T}\right)\right)^{N}$ and they are known and currently named interfering term, however, $\beta_{i} \in \mathbb{R}$ are small enough and unknown.

Without a loss of generality, we also assume that the functions $m_{i}$ are linearly independent in the algebraic sense.

Let us denote by $\mathcal{K}$, the finite vectorial dimensional subspace generated by the system $\left\{m_{i} \chi_{\omega_{T}}\right\}_{i=1, \cdots, M}$.

We are not interested in solving the problem (2) but denoting by $S_{0}(\lambda, \tau)=\int_{0}^{T} \int_{\mathcal{O}} h_{0} y(x, t ; \lambda, \tau) d x d t$ the global information provided by the observer $h_{0}$ in the observatory $\mathcal{O}$ during the interval $[0, T]$, our natural motivation consists in analyzing the insensitivity for $S_{0}(\lambda, \tau)$ with respect to the incomplete initial data and also the interferences. In others words, can we valid the following conditions:

$$
\frac{\partial S_{0}}{\partial \tau}(0,0)=0 ?
$$


and

$$
\int_{0}^{T} \int_{\mathcal{O}} h_{0} m_{i} d x d t=0, \forall i=1, \cdots, M ?
$$

Generally, these statements are not true therefore we cannot obtain some complete information on the pollution term without any sensibility respect to the missing data.

Here, to arise this problem, we plan to revisit the concept of sentinel due to J.L. Lions (for instance one can see [17] which is an introduction on the concept of sentinel and it has been done for some general evolutive system of partial differential equations). We propose to determinate of a function $\hat{w} \in\left(L^{2}\left(\omega_{T}\right)\right)^{N}$ acting on a sub-domain $\omega$ of $\mathcal{O}$. We set:

$$
\hat{\mathcal{S}}(\lambda, \tau)=\int_{0}^{T} \int_{\mathcal{O}} h_{0} y(x, t ; \lambda, \tau) d x d t+\int_{0}^{T} \int_{\omega} \hat{w} y(x, t ; \lambda, \tau) d x d t
$$

Then, firstly, the problem consists in looking for $\hat{w}$ such that the following conditions are satisfied

$\hat{\mathcal{S}}$ is insensitive at the first order to missing terms $\tau \hat{y}^{0}$ :

$$
\left.\frac{\partial \hat{\mathcal{S}}}{\partial \tau}(\lambda, \tau)\right|_{\lambda=0, \tau=0}=0, \quad \forall \hat{y^{0}}
$$

$\hat{\mathcal{S}}$ is insensitive to the interfering terms $\beta_{i} m_{i}$ :

$$
\int_{0}^{T} \int_{\mathcal{O}} h_{0} m_{i} d x d t+\int_{0}^{T} \int_{\omega} \hat{w} m_{i} d x d t=0 . \quad 1 \leq i \leq M
$$

Secondly, $\hat{w} \in\left(L^{2}\left(\omega_{T}\right)\right)^{N}$ is such that

$$
\frac{1}{2}\|\hat{w}\|_{L^{2}\left(\omega_{T}\right)}^{2}=\min _{w \in \hat{\mathcal{W}}} \frac{1}{2}\|w\|_{L^{2}\left(\omega_{T}\right)}^{2},
$$

where $\hat{\mathcal{W}}$ is the collection of $w$ satisfying to (5) and (6). Assuming that such a $\hat{w}$ exists, we have the following definition

Definition 0.1. Let $\hat{\mathcal{S}}$ be the real function defined by (4). $\hat{\mathcal{S}}$ is said to be a discriminating distributed sentinel defined by $h_{0}$ if there exists $\hat{w} \in\left(L^{2}\left(\omega_{T}\right)\right)^{N}$ such that properties (5)-(7) are valid.

Remark 0.2. We must notice that the existence of insensitive controls without constraints has been intensively studied for the heat equation and semi-linear in [1], [2], [13] and Stokes equations in [8] describing an linearized oceanic quasi-geostrophic model.

Our study is investigated under geometrical hypothesis. By contrary to [15], we suppose $\omega$ and $\mathcal{O}$ are not identical. More precisely, we assume $\omega \subset \mathcal{O}$. The next Remark follows.

Remark 0.3. Assume $\mathcal{O} \cap \omega=\emptyset$, then $\int_{0}^{T} \int_{\omega} w m_{i} d x d t=0$ thus, to satisfy (6), it is sufficient to choose $h_{0}$ orthogonal to each $m_{i}$. Now, assume $\mathcal{O} \subset \omega$, then (6) becomes $\int_{0}^{T} \int_{\mathcal{O}} h_{0} m_{i} d x d t+\int_{0}^{T} \int_{\mathcal{O}} w m_{i} d x d t=$ $0, \quad 1 \leq i \leq M$ which is the case of two identical domains discussed in [15]. Thus, without a loss of generality, we can assume that $\omega \subset \mathcal{O}$. 


\subsection{A null-controllability problem}

Now, let us prove that seeking for a control $\hat{w}$ such that (5)-(7) is equivalent to a null-controllability with constraints on the control. We proceed two steps.

Step1. We consider the functions $y_{0}$ and $p_{0}$ which solve problem (2) for $\lambda=0$ and $\tau=0$

$$
\left\{\begin{array}{rllll}
\frac{\partial y_{0}}{\partial t}-\Delta y_{0}+y_{0} \nabla y_{0}+\nabla p_{0} & = & & \text { in } & Q, \\
\text { divy } & = & 0 & \text { in } & Q, \\
y & = & 0 & \text { on } & \Sigma, \\
y_{\tau}(0) & = & y^{0} & \text { on } & \Omega .
\end{array}\right.
$$

Step 2. Assume that $\frac{\partial y}{\partial \tau}$ can be defined for $\lambda=\tau=0$. Then, the function $y_{\tau}=\frac{\partial y}{\partial \tau}(0,0)$ solves the problem

$$
\left\{\begin{array}{rllll}
\frac{\partial y_{\tau}}{\partial t}-\Delta y_{\tau}+\nabla\left(y_{\tau} \otimes y+y \otimes y_{\tau}\right)+\nabla p_{\tau} & = & 0 & \text { in } & Q, \\
d i v y_{\tau} & = & 0 & \text { in } & Q, \\
y_{\tau} & = & 0 & \text { on } & \Sigma, \\
y_{\tau}(0) & = & y^{0} & \text { on } & \Omega
\end{array}\right.
$$

(9) is a linear Navier-Stokes problem, more precisely this is a linearized form of (8) around $y_{0}$. Let $y_{\tau}$ its unique solution. If $y_{0}$ and $y_{\tau}$ solve respectively (8) and (9), then the insensibility condition (5) is equivalent to

$$
\int_{0}^{T} \int_{\mathcal{O}} h_{0} y_{\tau} d x d t+\int_{0}^{T} \int_{\omega} \hat{w} y_{\tau} d x d t=0, \quad \forall \hat{y}^{0}, \quad\left\|\hat{y}^{0}\right\|_{L^{2}(\Omega)} \leq 1 .
$$

We set $\mathcal{D} q=\nabla q+\nabla q^{t}$ and introduce the adjoint state system associated to (9)

$$
\left\{\begin{aligned}
-\frac{\partial q}{\partial t}-\Delta q-\mathcal{D} q y_{0}+\nabla \pi & =h_{0} \chi_{\mathcal{O}}+\hat{w} \chi \omega_{T} & & \text { in } \quad Q, \\
\operatorname{divq} & =0 & & \text { in } Q, \\
q & =0 & & \text { on } \Sigma, \\
q(T) & =0 & & \text { on } \Omega
\end{aligned}\right.
$$

Therefore, let $\hat{q}=q(\hat{w})$ be the unique solution, it is well known that $\hat{q} \in L^{2}\left(0, T ;\left(H_{0}^{1}(\Omega)\right)^{N}\right) \cap C^{0}\left([0, T] ;\left(L^{2}(\Omega)\right)^{N}\right)$ depends on $\hat{w}$ which is to be determined.

Moreover, if we multiply $(11)$ by $y_{\tau}$, after integrating by parts over $Q$, we obtain

$$
\int_{0}^{T} \int_{\mathcal{O}} h_{0} y_{\tau} d x d t+\int_{0}^{T} \int_{\omega} \hat{w} y_{\tau} d x d t=-\int_{\Omega} \hat{y}^{0} \hat{q}(0) d x, \quad \forall \hat{y}^{0}, \quad\left\|\hat{y}^{0}\right\|_{L^{2}(\Omega)} \leq 1 .
$$

This last equality combining with (10) becomes

$$
\int_{\Omega} \hat{y}^{0} \hat{q}(0) d x=0, \quad \forall \hat{y}^{0}, \quad\left\|\hat{y}^{0}\right\|_{L^{2}(\Omega)} \leq 1 .
$$

Consequently, insensibility condition (5) is valid if and only if

$$
\hat{q}(0)=0 \quad \text { in } \quad \Omega .
$$

In other words, condition (5) is satisfied if and only if we obtain a control $\hat{w}$ solution of the null-controllability problem (11)-(12) Now, let us modify the constraints (6). Indeed, since $\mathcal{K}$ is the vectorial subspace generated in $\left(L^{2}\left(\omega_{T}\right)\right)^{N}$ by the basis $\left(m_{1} \chi_{\omega_{T}}, \cdots, m_{M} \chi_{\omega_{T}}\right)$, there exists a unique $k_{0} \in \mathcal{K}$ such that 


$$
\int_{0}^{T} \int_{\mathcal{O}} h_{0} m_{i} d x d t+\int_{0}^{T} \int_{\omega} k_{0} m_{i} d x d t=0
$$

In other words, the condition (6) is equivalent to

$$
\hat{w}-k_{0}=\hat{k} \in \mathcal{K}^{\perp} .
$$

Finally, if we define the operator

whose adjoint is

$$
L=\frac{\partial}{\partial t}-\Delta+\mathcal{D}(I d) y_{0}
$$

here, $\mathcal{D}(I d) y_{0}$ is the linear operator defined as:

$$
L^{*}=-\frac{\partial}{\partial t}-\Delta+\mathcal{D}(I d) y_{0},
$$

$$
D(I d) y_{0}: q \longmapsto \mathcal{D} q y_{0}=\left(\nabla q+\nabla q^{t}\right) y_{0},
$$

it is well known that for $h$ and $k$ fixed respectively in $L^{2}(Q)^{N}$ and $L^{2}\left(\omega_{T}\right)^{N}$ the backward problem

$$
\left\{\begin{aligned}
L^{*} q+\nabla \pi & =h+k \chi_{\omega_{T}} & & \text { in } Q \\
\operatorname{divq} & =0 & & \text { in } Q \\
q & =0 & & \text { on } \Sigma \\
q(T) & =0 & & \text { in } \Omega
\end{aligned}\right.
$$

has a unique solution $q(k) \in\left(L^{2}(Q)\right)^{N}$ defined by transposition in the following sense

$$
\int_{Q} q(k) L \Psi d x d t=-\int_{Q}\left(h+k \chi_{\omega_{T}}\right) \Psi d x d t,
$$

$\forall \Psi$ such that $\operatorname{div} \Psi=0$ in $\left.\mathrm{Q} \Psi\right|_{\Sigma}=0$ and $\Psi(x, 0)=0$ in $\Omega$.

Consequently, the problem which consists in searching for a control $\hat{w}$ satisfying (5)-(7) is equivalent to look for $\hat{k}$ such that $(\hat{k}, \hat{q}) \in \mathcal{K}^{\perp} \times L^{2}\left(0, T ; L^{2}(\Omega)^{N}\right)$ is solution of the null-controllability problem subject to constraints.

and

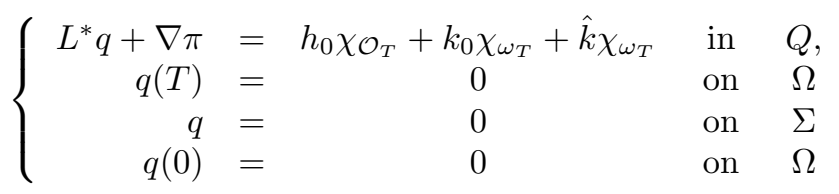

where

$$
\frac{1}{2}|\hat{k}|_{L^{2}\left(\omega_{T}\right)}=\min _{k \in \mathcal{E}} \frac{1}{2}|k|_{L^{2}\left(\omega_{T}\right)}
$$

$$
\mathcal{E}=\left\{k \in L^{2}\left(\omega_{T}\right) ; k \in \mathcal{K}^{\perp}, q(k)(., 0)=0 \text { in } \Omega\right\} .
$$

Then, solving (16) amounts in searching $\{\hat{k}, \hat{q}, \hat{\pi}\}$ with

$$
\hat{k} \in \mathcal{K}^{\perp}, \hat{q}=q(\hat{k}), \hat{\pi}=\pi(\hat{k})
$$

and

$$
\hat{q}(x, 0)=0 \quad \text { in } \quad \Omega .
$$

Furthermore, $\hat{k}$ requires the condition (17)-(18). 
Remark 0.4. Property (19) defines the set of constraints on $k$. Hence, problem (15)-(20) and (16) is a null-controllability type problem with constraints. When $\mathcal{K}=\left(L^{2}\left(\omega_{T}\right)\right)^{N}$, we notice that we have again a null-controllability problem without constraints on the control.

\subsection{Related studies}

During the nineties, the controllability for Navier-Stokes equations are intensively studied. Without constraints on the control, that means when $\mathcal{K}=\left(L^{2}\left(\omega_{T}\right)\right)^{N}$, it is now well know that Navier-Stokes equations locally null controllable (see, for instance [5], [6], [9], [10], [11], [12], [19]). The main existence results of a local control are based essentially on Carleman and observability inequalities.

In [15], using the Hilbert Uniqueness Method (H.U.M) introduced in [14], the author deals with $(2)$ when $\mathcal{K}$ is not $\left(L^{2}\left(\omega_{T}\right)\right)^{N}$, therefore the functions $h$ and $\hat{w}$ have the same support, that is $\mathcal{O}=\omega$.

More recently, for heat equation, seen in [20], the author uses an adapted Carleman inequality deriving global Carleman inequality and prove explicitly the existence of a constrained control when $\mathcal{K} \neq\left(L^{2}\left(\omega_{T}\right)\right)^{N}$ and $\omega \subset \mathcal{O}$ strictly instead of considering the situation $\mathcal{O}=\omega$. In this study, the author revisits the results obtained in [15]. In our paper, we deal with (15)-(20) and (16) when $\hat{k}$ and $h$ do not have necessary the same support and $\mathcal{K}$ is $\operatorname{not}\left(L^{2}\left(\omega_{T}\right)\right)^{N}$.

To present our results, we divide the study in four sections.

The first section is devoted to introduce the problem.

The second section deals with a general boundary null-controllability problem with constraints on the control. To do so, employing a Carleman Inequality for linear Navier-Stokes equations due to [9], we establish an Adapted Observability Inequality for Navier-Stokes problem with constraints.

The third section consists in giving a suitable characterization of the control obtained in the previous section. In the last section, we show how results obtained in previous sections on null-controllability problem with constraints can be applied to formulate the corresponding sentinel.

\subsection{Notations and Assumptions}

\subsubsection{Notations}

Throughout this paper, we adopt some notations and conventions.

We consider the sets:

$$
\mathcal{V}=\left\{(\rho, \pi) \in\left(C^{\infty}(\bar{Q})\right)^{N} \times C^{1}(\bar{Q}) ; \operatorname{div} \rho=0 \text { on } \mathrm{Q}, \int_{\omega_{T}} \pi d x d t=0 \text { and } \rho=0 \text { on } \Sigma\right\}
$$

and

$$
\mathcal{V}_{0}=\left\{(\rho, 0) ; \rho \in\left(C^{\infty}(\bar{Q})\right)^{N} ; \operatorname{div} \rho=0 \text { on } \mathrm{Q}, \quad \text { and } \rho=0 \text { on } \Sigma\right\} .
$$

We design by $P$ the orthogonal projection operator from $L^{2}\left(\omega_{T}\right)$ to $\mathcal{K}$. For any $\rho \in \mathcal{V}$, we shall denote by $P \rho \chi_{\omega_{T}}$ the projection into $\mathcal{K}$ of $\rho \chi_{\omega_{T}} \in L^{2}\left(\omega_{T}\right)$.

\subsubsection{Assumptions}

In this paper we assume the following hypotheses:

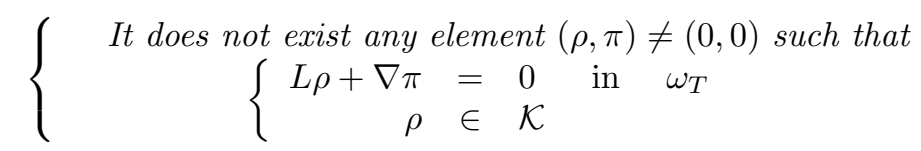

Remark 0.5. Assumption (21) seems natural (for instance, see [15] where similar hypothesis has been make). 
A geometrical assumption

$$
\omega \subset \Omega
$$

\subsection{Main results}

Our main results are summarized in the following theorems

Theorem 0.6. Assume (21). $h \in L^{2}(\mathcal{O})$ is a given function. There exists a system $\{\hat{k}, \hat{q}, \hat{\pi}\}$ satisfying (16)(18).

Theorem 0.7. The system $\{\hat{k}, \hat{q}, \hat{\pi}\}$ is defined as

$$
\hat{k}=P \hat{\rho} \chi_{\omega_{T}}-\hat{\rho} \chi_{\omega_{T}} \in \mathcal{K}^{\perp}
$$

where

$$
\begin{gathered}
\left\{\begin{array}{llllll}
L \hat{\rho} & = & 0 & \text { in } & Q \\
\operatorname{div} \hat{\rho} & 0 & \text { in } & Q \\
\hat{\rho} & = & 0 & \text { on } & \Sigma .
\end{array}\right. \\
\left\{\begin{array}{lllll}
L^{*} \hat{q}+\nabla \hat{\pi} & = & h+\hat{k} \chi_{\omega_{T}} & \text { in } & Q \\
\operatorname{div} \hat{q} & = & 0 & \text { in } & Q \\
\hat{q} & = & 0 & \text { on } & \Sigma \\
\hat{q}(T) & = & 0 & \text { in } & \Omega \\
\hat{q}(0) & = & 0 & \text { in } & \Omega .
\end{array}\right.
\end{gathered}
$$

A direct application to the theory of sentinel is made and we have:

Theorem 0.8. Let $\Omega$ be a bounded open set of class $C^{2}$ with a sufficiently smooth boundary $\Gamma$. Let $\omega$ a open subset of $\Omega$. $(\zeta, \hat{\zeta})$ and $\left(y^{0}, \hat{y}^{0}\right)$ belong in $\left(\left(L^{2}(Q)\right)^{N}\right)^{2}$ and $\left(\left(L^{2}(\Omega)\right)^{N}\right)^{2}$ respectively. Assume $\omega \subset \mathcal{O}$. Let $y$ be the unique solution of (2).

There exists a control $\hat{w} \in L^{2}\left(\omega_{T}\right)$ such that the function $\hat{\mathcal{S}}$ defined as

$$
\hat{\mathcal{S}}(\lambda, \tau ; \hat{w})=\int_{0}^{T} \int_{\mathcal{O}} h_{0} y(x, \lambda, \tau) d x d t+\int_{0}^{T} \int_{\omega} \hat{w} y(x, \lambda, \tau) d x d t
$$

is a Sentinel in the sense of Definition 0.1.

Moreover, let $\hat{q}\left(h_{0}\right)$ be the unique solution of (25) depending of $h_{0}, m_{0}$ and $y_{0}$ are defined as in (3) and (8) respectively, the pollution term is identified as follows:

$$
\int_{0}^{T} \int_{\Omega} \hat{q}\left(h_{0}\right)\{\lambda \hat{\xi}\} d x d t=\int_{0}^{T} \int_{\Omega}\left(h_{0} \chi_{\mathcal{O}}+\hat{w} \chi_{\omega}\right)\left(m_{0}-y_{0}\right) d x d t
$$

\section{Proof of Theorem 0.6. Existence of A CONTrol}

\subsection{An adapted Observability Inequality}

In this paper, the main tool used consists in an adapted inequality observability. Before presenting it, in a Lemma, we need to recall a global Carleman inequality obtained by [9] for the following linear Navier-Stokes system

$$
\left\{\begin{array}{rllll}
-\frac{\partial \phi}{\partial t}-\Delta \phi-D \phi \bar{y}+\nabla \pi & = & & \text { in } & Q \\
\operatorname{div} \phi & = & 0 & \text { in } & Q \\
\phi & = & 0 & \text { on } & \Sigma \\
\phi(T) & = & \phi^{0} & \text { on } & \Omega
\end{array}\right.
$$


where $\bar{y}$ is the solution of the uncontrolled Navier-stokes system

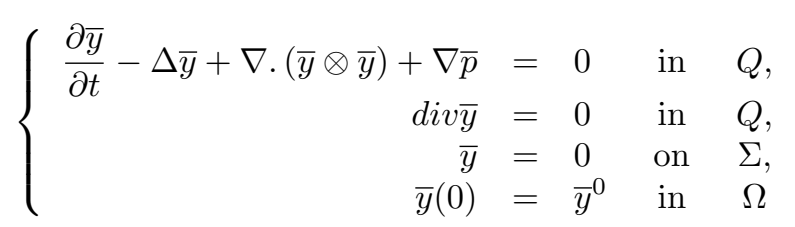

Lemma 1.1. Let us suppose that $\bar{y} \in\left(L^{\infty}(Q)\right)^{N}, \frac{\partial \bar{y}}{\partial t} \in\left(L^{2}\left(0, T ; L^{\sigma}(\Omega)\right)\right)^{N}, \sigma>6 / 5$ if $N=3, \sigma>1$ if $N=2$ hold. Then, there exist three positive constants $\hat{s}, \hat{D}, C$ depending on $\Omega$ and $\omega$ such that, for every $\phi^{0} \in\left\{y \in\left(L^{2}(\Omega)\right)^{N}\right.$

$\nabla \cdot y=0$ in $\Omega, y . n=0$ on $\partial \Omega\}$ and $g \in\left(L^{2}(Q)\right)^{N}$, the corresponding solution to (26) verifies:

$$
\begin{aligned}
& s^{3} D^{4} \int_{Q} e^{-2 s \alpha} \xi^{3}|\phi|^{2} d x d t+s D^{2} \int_{Q} e^{-2 s \alpha} \xi|\nabla \phi|^{2} d x d t \\
& +s^{-1} \int_{Q} e^{-2 s \alpha} \xi^{-1}\left(\left|\phi_{t}\right|^{2}+|\Delta \phi|^{2}\right) d x d t \\
& \leq C\left(1+T^{2}\right)\left(s^{15 / 2} D^{20} \int_{Q} e^{-4 s \hat{\alpha}+2 s \alpha^{*}} \xi^{15 / 2}|g|^{2} d x d t\right. \\
& \left.+s^{16} D^{40} \int_{\omega_{T}} e^{-8 s \hat{\alpha}+6 s \alpha^{*}} \xi^{16}|\phi|^{2} d x d t\right) .
\end{aligned}
$$

for all $D \geq \hat{D}\left(1+\|\bar{y}\|_{\infty}+\left\|\bar{y}_{t}\right\|_{L^{2}\left(0, T ; L^{\sigma}(\Omega)^{N}\right)}^{2}+e^{\hat{D} T\|\bar{y}\|_{\infty}}\right)$ and $s \geq \hat{s}\left(T^{4}+T^{8}\right)$ and appropriate positive weight functions $\alpha, \xi, \hat{\alpha}, \alpha^{*}, \hat{\xi}$.

Proof. (See [9])

The next Lemma is devoted to an Adapted Observability Inequality :

Lemma 1.2. There exists a constant $C>0$ depending on $\Omega, \omega_{0}, T$ such that for every $(\rho, \pi) \in \mathcal{V}$

$$
\int_{Q} \frac{1}{\Theta}|\rho|^{2} d x d t \leq C\left[\int_{Q}|L \rho+\nabla \pi|^{2} d x d t+\int_{\omega_{T}}\left|\rho \chi_{\omega_{T}}-P \rho \chi_{\omega_{T}}\right|^{2} d x d t\right]
$$

where $\Theta=s^{3} D^{4} e^{-2 s \alpha} \xi^{3}$.

Before starting the proof, let us notice the following remark

Remark 1.3. We can observe that Lemma 1.2 remains valid on $\mathcal{V}_{0}$ defined as in the beginning of this section.

Proof. Proof of Lemma 1.2

We argue by opposite. Let $n$ be an integer, suppose there exists a sequence $\left(\rho_{n}, \pi_{n}\right)$ such that

$$
\int_{Q}\left|L \rho_{n}+\nabla \pi_{n}\right| d x d t+\int_{\omega_{T}}\left|\rho_{n} \chi_{\omega_{T}}-P \rho_{n} \chi_{\omega_{T}}\right|^{2} d x d t<\frac{1}{n} \int_{Q} \frac{1}{\Theta}\left|\rho_{n}\right|^{2} d x d t
$$

We show that the sequences $\rho_{n}$ and $\pi_{n}$ are bounded. Let us denote again by $\rho_{n}$ and $\pi_{n}$, the extracted subsequences, we establish their convergence. Hence, we show that a contradiction occurs.

To do so, the proof is divided in two steps.

Step 1 
1.1.1. $\rho_{n}$ and estimates

We can assume $\int_{Q} \frac{1}{\Theta}\left|\rho_{n}\right|^{2} d x d t=1$. Then, we deduce

$$
\int_{Q}\left|L \rho_{n}+\nabla \pi_{n}\right|^{2} d x d t \longrightarrow 0
$$

and

$$
\int_{\omega_{T}}\left|\rho_{n} \chi_{\omega_{T}}-P \rho_{n} \chi \omega_{T}\right|^{2} d x d t \longrightarrow 0 .
$$

Before continuing, notice that we can write

$$
\frac{1}{\Theta} P \rho_{n}=\frac{1}{\Theta}\left(P \rho_{n} \chi_{\omega_{T}}-\rho_{n} \chi_{\omega_{T}}\right)+\frac{1}{\Theta} \rho_{n} .
$$

Since we have $\int_{Q} \frac{1}{\Theta}\left|\rho_{n}\right|^{2} d x d t=1, \frac{1}{\Theta}$ being bounded in $Q$, using (31) there exists a constant $K>0$ such that

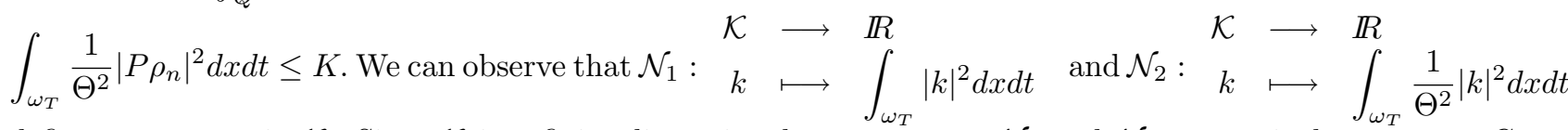
define two norms in $\mathcal{K}$. Since $\mathcal{K}$ is a finite dimensional vector space, $\mathcal{N}_{1}$ and $\mathcal{N}_{2}$ are equivalent norms. Consequently, doing $k=P \rho_{n}$, it follows $\int_{\omega_{T}}\left|P \rho_{n}\right|^{2} d x d t \leq K$. Hence, since the Pythagore identity, $\left\|\rho_{n} \chi_{\omega_{T}}\right\|_{\omega_{T}}^{2}=$ $\left\|\rho_{n} \chi_{\omega_{T}}-P \rho_{n} \chi_{\omega_{T}}\right\|_{\omega_{T}}^{2}+\left\|P \rho_{n} \chi_{\omega_{T}}\right\|_{\omega_{T}}^{2}$ we also have

$$
\int_{\omega_{T}}\left|\rho_{n} \chi_{\omega_{T}}\right|^{2} d x d t \leq K
$$

\subsection{2. $\rho_{n}$ and convergence}

From (32), we can extract a subsequence denoted again $\rho_{n}$ and so we can consider that $\rho_{n} \chi_{\omega_{T}}$ converges weakly in $\left(L^{2}\left(\omega_{T}\right)\right)^{N}$. There exists $\varphi \in\left(L^{2}\left(\omega_{T}\right)\right)^{N}$ such that

$$
\rho_{n} \chi_{\omega_{T}} \rightarrow \varphi \text { in }\left(L^{2}\left(\omega_{T}\right)\right)^{N}
$$

From compactness properties of operator $P$, it results that

$$
P \rho_{n} \chi_{\omega_{T}} \rightarrow P \varphi \text { in }\left(L^{2}\left(\omega_{T}\right)\right)^{N}
$$

Moreover, combining (31), (33) and (34), we have $\varphi=P \varphi$, and

$$
\rho_{n} \chi_{\omega_{T}}-\varphi \longrightarrow 0 \text { in }\left(L^{2}\left(\omega_{T}\right)\right)^{N}
$$

and that means

$$
\varphi \in \mathcal{K}
$$

We can deduce that $\rho_{n} \chi_{\omega_{T}} \longrightarrow \varphi$ in $\left(\mathcal{D}^{\prime}\left(\omega_{T}\right)\right)^{N}$ and so

$$
L \rho_{n} \chi_{\omega_{T}} \longrightarrow L \varphi \quad \text { in }\left(\mathcal{D}^{\prime}\left(\omega_{t}\right)\right)^{N}
$$

Step $2 \pi_{n}$ some estimates and convergence. 


\subsection{3. $\pi_{n}$ and estimates} that

Before starting, we must notice that (30) implies that there exits $\varepsilon_{n} \in\left(L^{2}(Q)\right)^{N}$ with $\left\|\varepsilon_{n}\right\|_{L^{2}(Q)} \rightarrow 0$ such

$$
\left\{\begin{array}{rlll}
L \rho_{n}+\nabla \pi_{n} & =\varepsilon_{n} & \text { in } & Q \\
\rho_{n} & =0 & \text { on } & \Sigma \\
\operatorname{div} \rho_{n} & =0 & \text { in } & Q \\
\rho_{n}(0) & =0 & \text { in } & \Omega
\end{array}\right.
$$

Inspired by ideas of [9], we introduce the sequences

$$
\begin{aligned}
\rho_{n}^{*} & =s^{1 / 4} e^{-s \alpha^{*}}\left(\xi^{*}\right)^{1 / 4} \rho_{n} \\
\pi_{n}^{*} & =s^{1 / 4} e^{-s \alpha^{*}}\left(\xi^{*}\right)^{1 / 4} \pi_{n} \\
g_{n}^{*} & =s^{1 / 4} e^{-s \alpha^{*}}\left(\xi^{*}\right)^{1 / 4} \varepsilon_{n}+s^{1 / 4} e^{-s \alpha^{*}}\left(\xi^{*}\right)^{1 / 4} D \rho_{n} \bar{y}-s^{1 / 4}\left(e^{-s \alpha^{*}}\left(\xi^{*}\right)^{1 / 4}\right)_{t} \rho_{n} .
\end{aligned}
$$

Then, the system (38) becomes a Stokes system

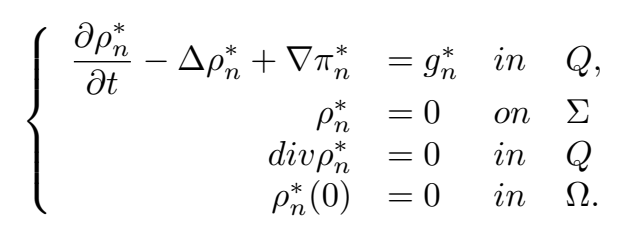

Thus, obviously we have $\rho_{n}^{*} \in L^{2}\left(O, T ; H^{2}(\Omega)^{N} \bigcap H_{0}^{1}(\Omega)^{N}\right) \bigcap L^{\infty}\left(O, T ; H_{0}^{1}(\Omega)^{N}\right)$ and $\pi_{n}^{*} \in L^{2}\left(O, T ; H^{1}(\Omega)\right.$ by the use of the regularity properties of evolutive Stokes equation (see for instance [22]). Furthermore there exists a positive constant $C$ such that

$$
\int_{Q}\left(\left|\pi_{n}^{*}\right|^{2}+\left|\nabla \pi_{n}^{*}\right|^{2}\right) d x d t \leq C \int_{Q}\left|g_{n}^{*}\right|^{2} d x d t
$$

However,

$$
\begin{aligned}
\int_{Q}\left|g_{n}^{*}\right|^{2} d x d t & \leq C s^{1 / 2}\left[\int_{Q} e^{-2 s \alpha^{*}}\left(\xi^{*}\right)^{1 / 2}\left|\varepsilon_{n}\right|^{2} d x d t+\int_{Q}\|\bar{y}\|_{\infty}^{2} e^{-2 s \alpha^{*}}\left(\xi^{*}\right)^{1 / 2}\left|\nabla \rho_{n}\right|^{2} d x d t\right. \\
& \left.+\int_{Q}\left|\left(e^{-s \alpha^{*}}\left(\xi^{*}\right)^{1 / 4}\right) t\right|^{2}\left|\rho_{n}\right|^{2} d x d t\right]
\end{aligned}
$$

Remembering that $\alpha^{*}(t)=\left(e^{5 / 4 \lambda m\left\|\nu^{0}\right\|_{\infty}}-e^{\lambda m\left\|\nu^{0}\right\|_{\infty}}\right) / t^{4}(T-t)^{4}$, and

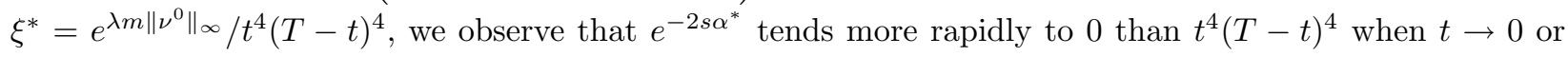
$t \rightarrow T$.

Consequently, we obtain

$$
\begin{aligned}
\int_{Q} s^{1 / 2} e^{-2 s \alpha^{*}}(\xi)^{1 / 2}\left|\pi_{n}\right|^{2} d x d t & \leq \int_{Q}\left|\varepsilon_{n}\right|^{2} d x d t+s D^{2} \int_{Q} e^{-2 s \alpha} \xi\left|\nabla \rho_{n}\right|^{2} d x d t \\
& +s^{3} D^{4} \int_{Q} e^{-2 s \alpha} \xi^{3}\left|\rho_{n}\right|^{2} d x d t
\end{aligned}
$$

However, on other hand, we observe that in the right hand of Carleman Inequality given in Theorem 1 [9], the weight functions $e^{-4 s \hat{\alpha}+2 s \alpha^{*}} \hat{\xi}^{15 / 2}$ and $e^{-8 s \hat{\alpha}+6 s \alpha^{*}} \hat{\xi}^{16}$ are bounded uniformly on $Q$ and $\omega_{T}$ respectively. Indeed, from definitions of $\hat{\alpha}, \alpha^{*}$, easy computation shows that

$$
-4 \hat{\alpha}+2 \alpha^{*}=-e^{5 / 4 m D}+2 e^{(m+1) D}-e^{m D} \text { and }-8 \hat{\alpha}+6 \alpha^{*}=-2 e^{5 / 4 m D}+8 e^{(m+1) D}-6 e^{m D} .
$$


Since $m$ is such that $m>4$, then for $D$ sufficiently large, $-4 \hat{\alpha}+2 \alpha^{*}$ and $-8 \hat{\alpha}+6 \alpha^{*}$ can be substituted by $-e^{5 / 4 m D}$ and $-2 e^{5 / 4 m D}$. Consequently, the weight functions $e^{-4 s \hat{\alpha}+2 s \alpha^{*}} \hat{\xi}^{15 / 2}$ and $e^{-8 s \hat{\alpha}+6 s \alpha^{*} \hat{\xi}^{16}}$ can be estimated respectively by

$$
e^{-s e^{\{5 / 4 m D\}} / t^{4}(T-t)^{4}} \frac{1}{t^{4}(T-t)^{4}} \text { and } e^{-2 s e^{\{5 / 4 m D\}} / t^{4}(T-t)^{4}} \frac{1}{t^{4}(T-t)^{4}} \text {. }
$$

We observe that when $t$ tends to 0 or $T$, then $e^{-s e^{\{5 / 4 m \lambda\}} / t^{4}(T-t)^{4}}$ and $e^{-2 s e^{\{5 / 4 m \lambda\}} / t^{4}(T-t)^{4}}$ tend more rapidly to 0 than $t^{4}(T-t)^{4}$.

It results

$$
\begin{aligned}
& s^{3} D^{4} \int_{Q} e^{-2 s \alpha} \xi^{3}\left|\rho_{n}\right|^{2} d x d t+s D^{2} \int_{Q} e^{-2 s \alpha} \xi\left|\nabla \rho_{n}\right|^{2} d x d t \\
& \leq C\left(\int_{Q}\left|\varepsilon_{n}\right|^{2} d x d t+\int_{\omega_{T}}\left|\rho_{n}\right|^{2} d x d t\right) .
\end{aligned}
$$

Combining (41) and (42), we have

$$
\int_{Q} s^{1 / 2} e^{-2 s \alpha^{*}}(\xi)^{1 / 2}\left|\pi_{n}\right|^{2} d x d t \leq C\left(\int_{Q}\left|\varepsilon_{n}\right|^{2} d x d t+\int_{\omega_{T}}\left|\rho_{n}\right|^{2} d x d t\right) .
$$

Since (40) we have

$$
\int_{Q}\left|\nabla \pi_{n}^{*}\right|^{2} d x d t \leq \int_{Q}\left|g_{n}^{*}\right|^{2} d x d t
$$

after adding (43) and (40), we get

$$
\begin{aligned}
\int_{Q}\left|\pi_{n}^{*}\right|^{2} d x d t+\int_{Q}\left|\nabla \pi_{n}^{*}\right|^{2} d x d t & \leq C\left(\int_{Q}\left|\varepsilon_{n}\right|^{2} d x d t+\int_{\omega_{T}}\left|\rho_{n}\right|^{2} d x d t\right) \\
& \leq C\left(\int_{Q}\left|L \rho_{n}+\nabla \rho_{n}\right|^{2} d x d t+\int_{\omega_{T}}\left|\rho_{n}\right|^{2} d x d t\right) .
\end{aligned}
$$

\subsection{4. $\pi_{n}$ and convergence}

We have assumed (29), then we deduce that $\pi_{n}^{*}$ is bounded in $L^{2}\left(10, T\left[\times H^{1}(\Omega)\right)\right.$. We can extract a subsequence denoted again $\pi_{n}^{*}$ converging weakly in $L^{2}(] 0, T\left[\times H^{1}(\Omega)\right)$. Let $\pi^{*}$ in $L^{2}(] 0, T\left[\times H^{1}(\Omega)\right)$ such that $\pi_{n}^{*} \rightarrow \pi^{*}$ in $L^{2}(] 0, T\left[\times H^{1}(\Omega)\right)$ then, $\pi_{n}^{*} \rightarrow \pi^{*}$ in $L^{2}(Q)$ and $\nabla \pi_{n}^{*} \rightarrow \nabla \pi^{*}$ in $\left(L^{2}(Q)\right)^{N}$.

Setting $\pi=s^{-1 / 4} e^{s \alpha^{*}}\left(\xi^{*}\right)^{-1 / 4} \pi^{*}$, we deduce that $\nabla \pi_{n} \rightarrow \nabla \pi$ in $\left(L^{2}(Q)\right)^{N}$, it also results that $\nabla \pi_{n} \rightarrow \nabla \pi$ in $\left(L^{2}\left(\omega_{T}\right)\right)^{N}$.

Using (37), we conclude that

$L \rho_{n} \chi_{\omega_{T}}+\nabla \pi_{n} \rightarrow L \varphi+\nabla \pi$ in $\left(L^{2}\left(\omega_{T}\right)\right)^{N}$.

Then, from (30), we deduce that

$$
L \varphi+\nabla \pi=0 \quad \text { in } \omega_{T} .
$$

From hypothesis $(21)$, we conclude that $\varphi=0$ in $\omega_{T}$. So, from $(35)$, it comes $\rho_{n} \longrightarrow 0$ in $\left(L^{2}\left(\omega_{T}\right)\right)^{N}$. Thanks to (30) and Theorem $1[9]$, we have $\int_{Q} e^{-2 s \alpha} \xi^{3}\left|\rho_{n}\right|^{2} d x d t \longrightarrow 0$. Assuming $\int_{Q} e^{-2 s \alpha} \xi^{3}\left|\rho_{n}\right|^{2} d x d t=1$, a contradiction occurs. Lemma 1.2 is proved.

\subsection{Existence of the best control.}

Let $(\rho, \pi),(\bar{\rho}, \bar{\pi})$ be in $\mathcal{V}$, we define

$$
a((\rho, \pi),(\bar{\rho}, \bar{\pi}))=\int_{Q}(L \rho+\nabla \pi)(L \bar{\rho}+\nabla \bar{\pi}) d x d t+\int_{\omega_{T}}(\rho-P \rho)(\bar{\rho}-P \bar{\rho}) d x d t .
$$


Lemma 1.4. The application $\begin{aligned} \mathcal{V} & \longrightarrow \mathbb{R}_{+} \\ (\rho, \pi) & \longmapsto \sqrt{a((\rho, \pi),(\rho, \pi))}\end{aligned}$ is a norm.

Proof. Particulary, we observe that $a((\rho, \pi),(\rho, \pi))=0$ implies simultaneously that $\int_{Q}|L \rho+\nabla \pi|^{2} d x d t=0$ and $\int_{\omega_{T}}\left|\rho \chi_{\omega_{T}}-P \rho \chi_{\omega_{T}}\right|^{2} d x d t=0$. That means, more precisely

$$
\left\{\begin{aligned}
L \rho \chi_{\omega_{T}}+\nabla \pi= & 0 \\
\rho \chi_{\omega_{T}} \in & \mathcal{K} .
\end{aligned}\right.
$$

From hypothesis $(21),(47)$ is valid if and only if $\rho=0$ in $\omega_{T}$. Using Lemma 1.2 or Theorem 1 [9], we deduce $\rho=0$ in $Q$. Introducing again the functions $\rho^{*}=s^{1 / 4} e^{-s \alpha^{*}} \xi^{1 / 4} \rho, \pi^{*}=s^{1 / 4} e^{-s \alpha^{*}} \xi^{1 / 4} \pi$, we have $\int_{Q}\left(\left|\pi^{*}\right|^{2}+\left|\nabla \pi^{*}\right|^{2}\right) d x d t \leq C \int_{Q}\left|g^{*}\right|^{2} d x d t$, where $g^{*}=s^{1 / 4} e^{-s \alpha^{*}}\left(\xi^{*}\right)^{1 / 4} D \rho \bar{y}-s^{1 / 4}\left(e^{-s \alpha^{*}}\left(\xi^{*}\right)^{1 / 4}\right)_{t} \rho$ in $Q$ and consequently, we obtain then $\pi^{*}=0$ and $\nabla \pi^{*}=0$ in $Q$. We get $\pi=0$ in $Q$.

Remark 1.5. From Remark 1.3, consider

$$
a_{0}((\rho, 0),(\bar{\rho}, 0))=\int_{Q} L \rho L \bar{\rho} d x d t+\int_{\omega_{T}}(\rho-P \rho)(\bar{\rho}-P \bar{\rho}) d x d t
$$

the bilinear form on $\mathcal{V}_{0} \times \mathcal{V}_{0}$. Obviously, $(\rho, 0) \longmapsto a_{0}((\rho, 0),(\rho, 0))$ defines a norm designated as \|\|$_{\Theta, 0}$. We can define by $V_{0}$ the completion of $\mathcal{V}_{0}$ respect to the norm \|\|$_{\Theta, 0}$.

Consider the completed space $V$ of $\mathcal{V}$ with respect to the norm $\|(\rho, \pi)\|_{\Theta}^{2}=a((\rho, \pi),(\rho, \pi))$. $\Theta$ is as in Lemma 1 , let $h$ be given in $\left(L^{2}(Q)\right)^{N}$ such that $\Theta h$ belongs in $\left(L^{2}(Q)\right)^{N}$, then we claim that $(\bar{\rho}, \bar{\pi}) \longrightarrow \int_{Q} h \bar{\rho} d x d t$ is continuous from $V$ to $\mathbb{R}$. Indeed, we have $\left|\int_{Q} h \bar{\rho} d x d t\right| \leq\|h \Theta\|_{L^{2}(Q)}\left\|\frac{1}{\Theta} \bar{\rho}\right\|_{L^{2}(Q)}$. Employing Lemma 1.2 and definition of $\|(\bar{\rho}, \bar{\pi})\|_{\Theta}$, the result announced is obvious. Lax-Milgram Lemma can be applied, there exists $\left(\rho_{\Theta}, \pi_{\Theta}\right)$ in $V$ such that

$$
a\left(\left(\rho_{\Theta}, \pi_{\Theta}\right),(\bar{\rho}, \bar{\pi})\right)=\int_{Q} h \bar{\rho} d x d t, \forall(\bar{\rho}, \bar{\pi}) \in V .
$$

1.2.1. Construction of $(q, \pi, k)$ satisfying (15) and (19)

Proposition 1.6. Let $\Theta$ be the function defined as in Lemma 1.2. Let $\left(\rho_{\Theta}, \pi_{\Theta}\right) \in V$ be the unique solution of (48). Assume $h$ is a function such that $\Theta h \in L^{2}(Q)$. Then there exists a system $\left\{k_{\Theta}, q_{\Theta}, \pi_{\Theta}\right\}$ satisfying to (16).

Proof. From (48), for any $(\bar{\rho}, \bar{\pi}) \in V$

$$
\int_{Q}\left(L \rho_{\Theta}+\nabla \pi_{\Theta}\right)(L \bar{\rho}+\nabla \bar{\pi}) d x d t+\int_{\omega_{T}}\left(\rho_{\Theta}-P \rho_{\Theta}\right)(\bar{\rho}-P \bar{\rho}) d x d t=\int_{\mathcal{O}} h \bar{\rho} d x d t
$$

Hence, taking $\bar{\pi}=0$, we obtain:

$$
\int_{Q}\left(L \rho_{\Theta}+\nabla \pi_{\Theta}\right) L \bar{\rho} d x d t+\int_{\omega_{T}}\left(\rho_{\Theta}-P \rho_{\Theta}\right) \bar{\rho} d x d t=\int_{Q} h \bar{\rho} d x d t, \quad \forall(\bar{\rho}, 0) \in V_{0} .
$$

We set $q_{\Theta}=L \rho_{\Theta}+\nabla \pi_{\Theta}$, we can write

$$
\int_{Q} q_{\Theta} L \bar{\rho} d x d t=\int_{Q}\left[h-\left(\rho_{\Theta} \chi_{\omega_{T}}-P \rho_{\Theta} \chi_{\omega_{T}}\right) \chi_{\omega_{T}}\right] \bar{\rho} d x d t, \quad \forall(\bar{\rho}, 0) \in V_{0} .
$$


However, let $\bar{\rho}$ be in $\left(C^{\infty}(\bar{Q})\right)^{N}$, after integrating by part over $Q$, we have

$$
\begin{aligned}
\int_{Q} q_{\Theta} L \bar{\rho} d x d t= & \left(q_{\Theta}(0), \bar{\rho}(0)\right)_{L^{2}(\Omega)}-\left(q_{\Theta}(T), \bar{\rho}(T)\right)_{L^{2}(\Omega)} \\
& +\int_{Q} L^{*} q_{\Theta} \bar{\rho} d x d t+\int_{\Sigma} q_{\Theta} \frac{\partial \bar{\rho}}{\partial \nu} d \sigma-\int_{\Sigma} \frac{\partial q_{\Theta}}{\partial \nu} \bar{\rho} d \sigma
\end{aligned}
$$

We choose $\bar{\rho} \in\left(C_{c}^{\infty}(Q)\right)^{N}$ such that $\operatorname{div} \bar{\rho}=0$ in $Q$. From (50) and (51) it results

$$
\int_{Q}\left\{L^{*} q_{\Theta}-\left[h-\left(\rho_{\Theta} \chi_{\omega_{T}}-P \rho_{\Theta} \chi_{\omega_{T}}\right) 1_{\omega_{T}}\right]\right\} \bar{\rho} d x d t=0 .
$$

$L^{*} q_{f}-\left[h-\left(\rho_{\Theta} \chi_{\omega_{T}}-P \rho_{\Theta} \chi_{\omega_{T}}\right) \chi_{\omega_{T}}\right]$ belongs in $L^{2}(Q)$, then there exists a pair $\left(q^{*}, \pi^{* *}\right)$ such that $L^{*} q^{*}+\nabla \pi^{* *}=$ $L^{*} q_{f}-\left[h-\left(\rho_{\Theta} \chi_{\omega_{T}}-P \rho_{\Theta} \chi_{\omega_{T}}\right) \chi_{\omega_{T}}\right]$, in particular, we have $d i v q^{*}=0$ in $Q, q^{*}=0$ on $\Sigma$ and $\nabla \pi^{* *} \in L^{2}(Q)$. Multiplying by $\bar{\rho}$, after integrating over $Q$, we have $\int_{Q}\left(L^{*} q^{*}+\nabla \pi^{* *}\right) \bar{\rho} d x d t=0$. Since, we have $d i v \bar{\rho}=0$ in $Q$, it follows that $\int_{Q} L^{*} q^{*} \bar{\rho} d x d t=0$ and so we get $L^{*} q^{*}=0$ in $Q$.

Setting $\pi_{h, \Theta}=-\pi^{* *}$, we deduce that there exists $\pi_{h, \Theta}$ such that

$$
L^{*} q_{\Theta}+\nabla \pi_{h, \Theta}=h-\left(\rho_{\Theta} \chi_{\omega_{T}}-P \rho_{\Theta} \chi_{\omega_{T}}\right) \chi_{\omega_{T}}, \text { in } Q
$$

Now, we take $\bar{\rho} \in C^{\infty}(\bar{Q})$, such that $\bar{\rho}(0)=\bar{\rho}(T)=0$, combining $(52)$ and doing successively $\bar{\rho}(0)=\bar{\rho}(T)=0$ firstly, we obtain $q_{\Theta}=0$ on $\Sigma$ when $\bar{\rho}=0$ on $\Sigma$ and secondly, $\frac{\partial q_{\Theta}}{\partial \nu}=0$ on $\Sigma$ when $\frac{\partial \bar{\rho}}{\partial \nu}=0$. Using Lions-Magenes arguments [16], we can define $q_{\Theta}(0)$ and $q_{\Theta}(T)$. Taking $\bar{\rho}(0)=0$ respectively $\bar{\rho}(T)=0$, we get $q_{\Theta}(0)=0$ and $q_{\Theta}(T)=0$ in $\Omega$.

We show that $\nabla \cdot q_{\Theta}=0$ in $\mathcal{O}_{T}$. To prove it, we set $k_{\Theta}=-\left(\rho_{\Theta} \chi_{\omega_{T}}-P \rho_{\Theta} \chi_{\omega_{T}}\right)$ and we consider the unique weak solution $(\tilde{q}, \tilde{\pi})$ of linear Navier-Stokes problem

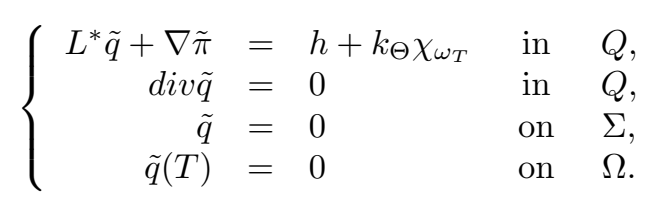

It is obvious to notice that $\tilde{q} \in\left(L^{2}(Q)\right)^{N}$ is the unique solution via transposition process in the following sense: for any $\psi \in\left(L^{2}(Q)\right)^{N}$,

$$
\int_{Q} \tilde{q} \psi d x d t=\int_{Q}\left(h+k_{\Theta} \chi_{\omega_{T}}\right) \rho_{\psi} d x d t
$$

where $\left(\rho_{\psi}, \pi_{\psi}\right)$ is the unique solution of the backward linear Navier-Stokes problem

$$
\left\{\begin{array}{rllll}
L \rho_{\psi}+\nabla \pi_{\psi} & = & \psi & \text { in } & Q \\
\operatorname{div} \rho_{\psi} & = & 0 & \text { in } & Q \\
\rho_{\psi} & = & 0 & \text { on } & \Sigma \\
\rho_{\psi}(0) & = & 0 & \text { on } & \Omega
\end{array}\right.
$$


We observe that $\left(\nabla \tilde{\pi}, \rho_{\psi}\right)_{L^{2}(Q)}=0$. Indeed,

$$
\begin{aligned}
\left(\nabla \tilde{\pi}, \rho_{\psi}\right)_{L^{2}(Q)} & =\sum_{i=1}^{N} \int_{Q} \frac{\partial \tilde{\pi}}{\partial x_{i}} \rho_{\psi_{i}} d x d t \\
& =\sum_{i=1}^{N} \int_{Q} \frac{\partial}{\partial x_{i}}\left(\tilde{\pi} \rho_{\psi_{i}}\right) d x d t-\int_{Q} \tilde{\pi} \sum_{i=1}^{N} \frac{\partial \rho_{\psi_{i}}}{\partial x_{i}} d x d t \\
& =\int_{\Sigma}^{\tilde{\pi}} \rho_{\psi} d \sigma d t-\int_{Q} \tilde{\pi} d i v \rho_{\psi} d x d t=0
\end{aligned}
$$

However, on other hand, since we have

$$
\int_{Q} q_{\Theta} \psi d x d t=\int_{Q} q_{\Theta}\left(L \rho_{\psi}+\nabla \pi_{\psi}\right) d x d t=\int_{Q}\left(L \rho_{\Theta}+\nabla \pi_{\Theta}\right)\left(L \rho_{\psi}+\nabla \pi_{\psi}\right) d x d t
$$

putting $\bar{\rho}=\rho_{\psi}$ and $\bar{\pi}=\pi_{\psi}$ in (49), we also conclude that

$$
\int_{Q} q_{\Theta} \psi d x d t=\int_{Q}\left(h+k_{\Theta} \chi_{\omega_{T}}\right) \rho_{\psi} d x d t
$$

The uniqueness argument implies that $q_{\Theta}=\tilde{q}$ in $Q$. So, we get by particulary, $\operatorname{div} q_{\Theta}=\operatorname{div} \tilde{q}=0$.

We have proved that there exists a control $k_{\Theta} \in \mathcal{K}, k_{\Theta}=P \rho_{\Theta} \chi_{\omega_{T}}-\rho_{\Theta} \chi_{\omega_{T}}$ such that $q\left(k_{\Theta}\right)=q_{\Theta}$ verifies

$$
\left\{\begin{aligned}
L^{*} q_{\Theta}+\nabla \pi_{h, \Theta} & =h+k_{\Theta} \chi_{\omega_{T}} & & \text { in } Q, \\
d i v q_{\Theta} & =0 & & \text { in } Q, \\
q_{\Theta} & =0 & & \text { on } \Sigma, \\
q_{\Theta}(0)=q_{\Theta}(T) & =0 & & \text { on } \Omega .
\end{aligned}\right.
$$

\subsubsection{Existence of $\hat{k}$}

$\mathcal{E}$ being as in (18), the previous section shows that $\mathcal{E}$ is non-empty. Moreover, $\mathcal{E}$ is convex and closed in $L^{2}\left(\omega_{T}\right)$. Consequently, the optimal problem $\min _{k \in \mathcal{E}} \frac{1}{2}\|k\|_{L^{2}\left(\omega_{T}\right)}^{2}$ admits a unique solution in $\mathcal{E}$ and so let us denote $\hat{k}$ its corresponding solution, $\hat{k}$ is unique. According to the structure of the set $\mathcal{E}$, there exists a pair $(\hat{q}, \hat{\pi})$ such that

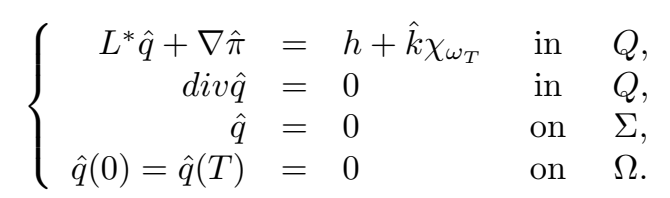

Now, we are interested in showing that there exists $\hat{\rho}$ such that $\hat{k}=P \hat{\rho} \chi_{\omega_{T}}-\hat{\rho} \chi_{\omega_{T}}$. We deal with this point in the next section.

\section{Proof of Theorem 0.7. A characterization of $\hat{k}$ Via an optimal system}

In order to characterize $\hat{k}$, we define the sets

$$
\mathcal{Q}=\left\{q ; L^{*} q \in\left(L^{2}(Q)\right)^{N}, \nabla \cdot q=0, q(T)=0, q(0)=0\right\},
$$




$$
\begin{gathered}
\mathcal{P}=\left\{\pi ; \nabla \pi \in\left(L^{2}(Q)\right)^{N}\right\}, \\
\left.\mathcal{U}=\left\{(k, q, \pi) ; k \in \mathcal{K}^{\perp},(q, \pi) \in \mathcal{Q} \times \mathcal{P}\right)\right\} .
\end{gathered}
$$

From the last section, we have $\left(q_{\Theta}, \pi_{\Theta}, k_{\Theta}\right) \in \mathcal{U}$, then $\mathcal{U}$ is a nonempty set.

Now, $\varepsilon>0$ is fixed, consider the function $J_{\varepsilon}$ defined on $\mathcal{U}$ by

$$
J_{\varepsilon}(k, q, \pi)=\frac{1}{2}\|k\|_{L^{2}\left(\omega_{T}\right)}^{2}+\frac{1}{2 \varepsilon}\left|L^{*} q+\nabla \pi-\left(h+k \chi_{\omega_{T}}\right)\right|_{L^{2}\left(\omega_{T}\right)}^{2} .
$$

$J_{\varepsilon}$ is positive, we set $d_{\varepsilon}=\inf _{(k, q, \pi) \in \mathcal{U}} J_{\varepsilon}(k, q, \pi)$. Let $\left.\left\{\left(k_{\varepsilon}^{n}, q_{\varepsilon}^{n}, \pi_{\varepsilon}^{n}\right)\right)\right\}_{n \in \mathbb{N}}$ a minimizing sequence such that

$$
d_{\varepsilon} \leq J_{\varepsilon}\left(k_{\varepsilon}^{n}, q_{\varepsilon}^{n}, \pi_{\varepsilon}^{n}\right) \leq d_{\varepsilon}+\frac{1}{n} .
$$

Proposition 2.1. Assume that $h \in\left(L^{2}(Q)\right)^{N}$ such that $\Theta h$ belongs to $\left(L^{2}(Q)\right)^{N}$. Then for all $0<\varepsilon<1$, there exists a unique system $\left(k_{\varepsilon}, q_{\varepsilon}, \pi_{\varepsilon}\right) \in \mathcal{U}$ such that

$$
J_{\varepsilon}\left(k_{\varepsilon}, q_{\varepsilon}, \pi_{\varepsilon}\right) \leq J_{\varepsilon}(k, q, \pi) \quad \forall(k, q, \pi) \in \mathcal{U} .
$$

Proof. 2.1. Convergence of the minimizing sequence $\left(k_{\varepsilon}^{n}, q_{\varepsilon}^{n}, \pi_{\varepsilon}^{n}\right)$

We establish this result following three steps

\subsubsection{Convergence of $k_{\varepsilon}^{n}$ and $q_{\varepsilon}^{n}$}

From properties of the sequence $\left(k_{\varepsilon}^{n}, q_{\varepsilon}^{n}, \pi_{\varepsilon}^{n}\right)$, we have

$$
\frac{1}{2}\left\|k_{\varepsilon}^{n}\right\|_{L^{2}\left(\omega_{T}\right)}^{2}+\frac{1}{2 \varepsilon}\left|L^{*} q_{\varepsilon}^{n}+\nabla \pi_{\varepsilon}^{n}-\left(h+k_{\varepsilon}^{n} \chi_{\omega_{T}}\right)\right|_{L^{2}(Q)}^{2} \leq d_{\varepsilon}+\frac{1}{n} .
$$

Since $\left(k_{\Theta}, q_{\Theta}, \pi_{\Theta}\right)$ is in $\mathcal{U}$, by particulary, we have:

$$
\frac{1}{2}\left\|k_{\varepsilon}^{n}\right\|_{L^{2}\left(\omega_{T}\right)}^{2}+\frac{1}{2 \varepsilon}\left|L^{*} q_{\varepsilon}^{n}+\nabla \pi_{\varepsilon}^{n}-\left(h+k_{\varepsilon}^{n} \chi_{\omega_{T}}\right)\right|_{L^{2}(Q)}^{2} \leq \frac{1}{2}\left\|k_{\Theta}\right\|_{L^{2}\left(\omega_{T}\right)}^{2}+1 .
$$

Consequently, we obtain successively:

$$
\left\|k_{\varepsilon}^{n}\right\|_{L^{2}\left(\omega_{T}\right)} \leq C \text { and }\left\|L^{*} q_{\varepsilon}^{n}+\nabla \pi_{\varepsilon}^{n}-\left(h+k_{\varepsilon}^{n} \chi_{\omega_{T}}\right)\right\|_{L^{2}(Q)} \leq C,
$$

where $C$ designates a constant not depending on $\varepsilon$ and $n$. We deduce that the sequences $k_{\varepsilon}^{n}, q_{\varepsilon}^{n}$ and $\pi_{\varepsilon}^{n}$ are weakly converging respectively. Indeed, since $\left|L^{*} q_{\varepsilon}^{n}+\nabla \pi_{\varepsilon}^{n}-\left(h+k_{\varepsilon}^{n} \chi \omega_{T}\right)\right| \leq C$ then there exists $h_{\varepsilon}^{n} \in B(0 ; C)$ such that $L^{*} q_{\varepsilon}^{n}+\nabla \pi_{\varepsilon}^{n}-\left(h+k_{\varepsilon}^{n} \chi_{\omega_{T}}\right)=h_{\varepsilon}^{n}$. So, we observe that the system $\left\{k_{\varepsilon}^{n}, q_{\varepsilon}^{n}, \pi_{\varepsilon}^{n}\right\}$ is such that

$$
\left\{\begin{aligned}
L^{*} q_{\varepsilon}^{n}+\nabla \pi_{\varepsilon}^{n} & =h+k_{\varepsilon}^{n} \chi_{\omega_{T}}+h_{\varepsilon}^{n} & & \text { in } Q, \\
\operatorname{div} q_{\varepsilon}^{n} & =0 & & \text { in } Q, \\
q_{\varepsilon}^{n} & =0 & & \text { on } \Sigma, \\
q_{\varepsilon}^{n}(0)=q_{\varepsilon}^{n}(T) & =0 & & \text { in } \Omega .
\end{aligned}\right.
$$

After multiplying (56) by $q_{\varepsilon}^{n}$ and integrating by part over $Q$, we obtain the following estimate:

$$
\begin{aligned}
& \frac{1}{2}\left\|q_{\varepsilon}^{n}(0)\right\|_{L^{2}(\Omega)}^{2}-\frac{1}{2}\left\|q_{\varepsilon}^{n}(T)\right\|_{L^{2}(\Omega)}^{2}+\left\|q_{\varepsilon}^{n}\right\|_{L^{2}\left(0, T ; H_{0}^{1}(\Omega)\right)}^{2}+\left(q_{\varepsilon}^{n}, \nabla \pi_{\varepsilon}^{n}\right) \\
& \leq\left(\left\|h+k_{\varepsilon}^{n} 1_{\omega_{T}}\right\|_{L^{2}(Q)}+\left\|h_{\varepsilon}^{n}\right\|_{L^{2}(Q)}\right)\left\|q_{\varepsilon}^{n}\right\|_{L^{2}(Q)} \\
& \leq\left(\left\|h+k_{\varepsilon}^{n} 1_{\omega_{T}}\right\|_{L^{2}(Q)}+\left\|h_{\varepsilon}^{n}\right\|_{L^{2}(Q)}\right)\left\|q_{\varepsilon}^{n}\right\|_{L^{2}\left(0, T ; H_{0}^{1}(\Omega)\right)} .
\end{aligned}
$$


As above, because $\operatorname{div} q_{\varepsilon}^{n}=0$ in $Q$, we can show that $\left(q_{\varepsilon}^{n}, \nabla \pi_{\varepsilon}^{n}\right)_{L^{2}\left(\mathcal{O}_{T}\right)}=0$. Then, since $q_{\varepsilon}^{n}(0)=q_{\varepsilon}^{n}(T)=0$, after simplifying, we see that $\left\|q_{\varepsilon}^{n}\right\|_{L^{2}\left(0, T ; H_{0}^{1}(\Omega)\right)} \leq C$. So, $q_{\varepsilon}^{n}$ converges weakly in $\left(L^{2}\left(0, T ; H_{0}^{1}(\Omega)\right)\right)^{N}$. Extracting a subsequence denoted again $q_{\varepsilon}^{n}$, we can suppose that $q_{\varepsilon}^{n}$ converges strongly in $\left(L^{2}(Q)\right)^{N}$. Let $q_{\varepsilon}$ its strong limit in $\left(L^{2}(Q)\right)^{N}$, this implies that $q_{\varepsilon}^{n}$ converges strongly to $q_{\varepsilon}$ in $\left(\mathcal{D}^{\prime}(Q)\right)^{N}$.

\subsection{2. $\pi_{\varepsilon}^{n}$ and convergence results}

We introduce again the sequences $q_{\varepsilon}^{* n}$ and $\pi_{\varepsilon}^{* n}$ defined as $q_{\varepsilon}^{* n}=s^{1 / 4} e^{-s \alpha^{*}}\left(\xi^{*}\right)^{1 / 4} q_{\varepsilon}^{n}$ and $\pi_{\varepsilon}^{* n}=s^{1 / 4} e^{-s \alpha^{*}}\left(\xi^{*}\right)^{1 / 4} \pi_{\varepsilon}^{n}$. Hence, (56) becomes

$$
\left\{\begin{array}{rllll}
L^{*} q_{\varepsilon}^{* n}+\nabla \pi_{\varepsilon}^{* n} & = & g_{\varepsilon}^{* n} & \text { in } & Q, \\
\operatorname{div} q_{\varepsilon}^{* n} & = & 0 & \text { in } & Q, \\
q_{\varepsilon}^{* n} & = & 0 & \text { on } & \Sigma, \\
q_{\varepsilon}^{* n}(0)=q_{\varepsilon}^{* n}(T) & = & 0 & \text { on } & \Omega .
\end{array}\right.
$$

where $g_{\varepsilon}^{* n}=s^{1 / 4} e^{-s \alpha^{*}}\left(\xi^{*}\right)^{1 / 4} g_{\varepsilon}^{n}+s^{1 / 4} e^{-s \alpha^{*}}\left(\xi^{*}\right)^{1 / 4} D q_{\varepsilon}^{n} \bar{y}-s^{1 / 4}\left(e^{-s \alpha^{*}}\left(\xi^{*}\right)^{1 / 4}\right)_{t} q_{\varepsilon}^{n}$, and $g_{\varepsilon}^{n}=h+k_{\varepsilon}^{n} \chi_{\omega_{T}}+h_{\varepsilon}^{n}$. According to properties of regularity for Stokes system, we have:

$$
\int_{Q}\left(\left|\pi_{\varepsilon}^{* n}\right|^{2}+\left|\nabla \pi_{\varepsilon}^{* n}\right|^{2}\right) d x d t \leq \int_{\mathcal{O}_{T}}\left|g_{\varepsilon}^{* n}\right|^{2} d x d t
$$

From above, we deduce that $\pi_{\varepsilon}^{* n}$ converges weakly in $\left(L^{2}\left(0, T ; H^{1}(\Omega)\right)\right)^{N}$. Let $\pi_{\varepsilon}^{*} \in\left(L^{2}\left(0, T ; H^{1}(\Omega)\right)\right)^{N}$ such that $\pi_{\varepsilon}^{* n} \rightarrow \pi_{\varepsilon}^{*}$ in $\left(L^{2}\left(0, T ; H^{1}(\Omega)\right)\right)^{N}$. In particular, by Rellich-Kondrachov Theorem, we also have $\pi_{\varepsilon}^{* n} \rightarrow \pi_{\varepsilon}^{*}$ in $\left(L^{2}(Q)\right)^{N}$. Remembering that $\pi_{\varepsilon}^{n}=s^{-1 / 4} e^{s \alpha^{*}}\left(\xi^{*}\right)^{-1 / 4} \pi_{\varepsilon}^{* n}$, we claim that $\pi_{\varepsilon}^{n}$ converges weakly in $\left(\mathcal{D}^{\prime}(Q)\right)^{N}$. Indeed, let $\psi \in \mathcal{D}(Q)$, we have:

$$
\left\langle\pi_{\varepsilon}^{n}, \psi\right\rangle=\left\langle s^{-1 / 4} e^{s \alpha^{*}}\left(\xi^{*}\right)^{-1 / 4} \pi_{\varepsilon}^{* n}, \psi\right\rangle=\left\langle\pi_{\varepsilon}^{* n}, s^{-1 / 4} e^{s \alpha^{*}}\left(\xi^{*}\right)^{-1 / 4} \psi\right\rangle .
$$

Setting $\psi^{*}=s^{-1 / 4} e^{s \alpha^{*}}\left(\xi^{*}\right)^{-1 / 4} \psi$, then $\psi^{*}$ remains in $(\mathcal{D}(Q))^{N}$ and moreover since $\pi_{\varepsilon}^{* n} \rightarrow \pi_{\varepsilon}^{*}$ in $\left(L^{2}(Q)\right)^{N}$, we get $\left\langle\pi_{\varepsilon}^{* n}, \psi^{*}\right\rangle \rightarrow\left\langle\pi_{\varepsilon}^{*}, \psi^{*}\right\rangle$. We also have $\left\langle\pi_{\varepsilon}^{*}, \psi^{*}\right\rangle=\left\langle s^{-1 / 4} e^{s \alpha^{*}}\left(\xi^{*}\right)^{-1 / 4} \pi_{\varepsilon}^{*}, \psi\right\rangle$.

We conclude that $\pi_{\varepsilon}^{n} \rightarrow s^{-1 / 4} e^{s \alpha^{*}}\left(\xi^{*}\right)^{-1 / 4} \pi_{\varepsilon}^{*}$ weakly in $\left(\mathcal{D}^{\prime}(Q)\right)^{N^{\varepsilon}}$. Throughout the sequel, we put $\pi_{\varepsilon}=$ $s^{-1 / 4} e^{s \alpha^{*}}\left(\xi^{*}\right)^{-1 / 4} \pi_{\varepsilon}^{*}$.

\subsection{3. $\nabla \pi_{\varepsilon}^{n}$ and convergence results}

Now, we show that $\nabla \pi_{\varepsilon}^{n} \rightarrow \nabla \pi_{\varepsilon}$ in $\left(\mathcal{D}^{\prime}(Q)\right)^{N}$. To do so, consider $\Psi=\left(\Psi_{i}\right)_{i=1 ; \cdots, N} \in(\mathcal{D}(Q))^{N}$. We have $\left\langle\frac{\partial \pi_{\varepsilon}^{n}}{\partial x_{i}}, \Psi_{i}\right\rangle=\left\langle\pi_{\varepsilon}^{n}, \frac{\partial \Psi_{\varepsilon}^{n}}{\partial x_{i}}\right\rangle$ for $i=1 ; \cdots ; N$. Since we have $\pi_{\varepsilon}^{n}=s^{-1 / 4} e^{s \alpha^{*}}\left(\xi^{*}\right)^{-1 / 4} \pi_{\varepsilon}^{* n}$, we can also write $\left\langle\frac{\partial \pi_{\varepsilon}^{n}}{\partial x_{i}}, \Psi_{i}\right\rangle=-\left\langle s^{-1 / 4} e^{s \alpha^{*}}\left(\xi^{*}\right)^{-1 / 4} \pi_{\varepsilon}^{* n}, \frac{\partial \Psi_{i}}{\partial x_{i}}\right\rangle$ for $i=1 ; \cdots ; N$. We also have $\left\langle s^{-1 / 4} e^{s \alpha^{*}}\left(\xi^{*}\right)^{-1 / 4} \pi_{\varepsilon}^{* n}, \frac{\partial \Psi_{i}}{\partial x_{i}}\right\rangle=$ $\left\langle\pi_{\varepsilon}^{* n}, s^{-1 / 4} e^{s \alpha^{*}}\left(\xi^{*}\right)^{-1 / 4} \frac{\partial \Psi_{i}}{\partial x_{i}}\right\rangle$ for $i=1 ; \cdots ; N$.

We deduce

$$
\begin{aligned}
\left\langle\pi_{\varepsilon}^{* n}, s^{-1 / 4} e^{s \alpha^{*}}\left(\xi^{*}\right)^{-1 / 4} \frac{\partial \Psi_{i}}{\partial x_{i}}\right\rangle & =\left\langle s^{-1 / 4} e^{s \alpha^{*}}\left(\xi^{*}\right)^{-1 / 4} \frac{\partial \pi_{\varepsilon}^{*}}{\partial x_{i}}, \Psi_{i}\right\rangle \\
& =\left\langle\frac{\partial \pi_{\varepsilon}}{\partial x_{i}}, \Psi_{i}\right\rangle .
\end{aligned}
$$

Since $\pi_{\varepsilon}^{* n} \rightarrow \pi_{\varepsilon}^{*}$ in $\left(L^{2}(Q)\right)^{N}$, then we conclude that for any $i=1 ; \cdots ; N$

$$
\left\langle\frac{\partial \pi_{\varepsilon}^{n}}{\partial x_{i}}, \Psi_{i}\right\rangle \rightarrow\left\langle\frac{\partial \pi_{\varepsilon}}{\partial x_{i}}, \Psi_{i}\right\rangle
$$


That means that $\nabla \pi_{\varepsilon}^{n} \rightarrow \nabla \pi_{\varepsilon}$ in $\left(\mathcal{D}^{\prime}(Q)\right)^{N}$.

It results that $h_{\varepsilon}^{n} \rightarrow L^{*} q_{\varepsilon}+\nabla \pi_{\varepsilon}-\left(h+k_{\varepsilon} \chi_{\omega_{T}}\right)$ in $\left(\mathcal{D}^{\prime}(Q)\right)^{N}$. However, $h_{\varepsilon}^{n}$ converges weakly in $\left(L^{2}(Q)\right)^{N}$, then from uniqueness, we deduce that $L^{*} q_{\varepsilon}+\nabla \pi_{\varepsilon}-\left(h+k_{\varepsilon} \chi_{\omega_{T}}\right) \in\left(L^{2}(Q)\right)^{N}$.

We achieve this part in showing that $\nabla \cdot q_{\varepsilon}=0$, in $Q q_{\varepsilon}(O)=q_{\varepsilon}(T)=0$ in $L^{2}(\Omega)$. Indeed, for any $\psi \in(\mathcal{D}(Q))^{N}$, we have:

$$
\int_{Q}\left(d i v q_{\varepsilon}^{n}\right) \psi d x d t=-\int_{Q} q_{\varepsilon}^{n} d i v \psi d x d t .
$$

Since $q_{\varepsilon}^{n} \rightarrow q_{\varepsilon}$ in $\left(L^{2}(Q)\right)^{N}$, we have $\int_{Q}\left(d i v q_{\varepsilon}^{n}\right) \psi d x d t \longrightarrow-\int_{Q} q_{\varepsilon} d i v \psi d x d t$. Consequently, since we have $\int_{Q} q_{\varepsilon} d i v \psi d x d t=-\int_{Q}\left(\operatorname{div} q_{\varepsilon}\right) \psi d x d t$ and $d i v q_{\varepsilon}^{n}=0$, we conclude that $\int_{Q}\left(\operatorname{div} q_{\varepsilon}\right) \psi d x d t=0$ and so div $q_{\varepsilon}=0$ in $Q$.

\subsection{Some a priori estimates on the sequence $\left(k_{\varepsilon}, q_{\varepsilon}, \pi_{\varepsilon}\right)$.}

Before dealing with this part, we observe, from the previous paragraph, that $\left(k_{\varepsilon}, q_{\varepsilon}, \pi_{\varepsilon}\right) \in \mathcal{U}$. Now, we pass to the low limit in (55). On one hand, as mentioned above, we obtain:

$$
\frac{1}{2}\left\|k_{\varepsilon}\right\|_{L^{2}\left(\omega_{T}\right)}^{2}+\frac{1}{2 \varepsilon}\left\|L^{*} q_{\varepsilon}+\nabla \pi_{\varepsilon}-\left(h+k_{\varepsilon} \chi_{\omega_{T}}\right)\right\|_{L^{2}(Q)}^{2} \leq \liminf _{n \rightarrow+\infty} J_{\varepsilon}\left(k_{\varepsilon}^{n}, q_{\varepsilon}^{n}, \pi_{\varepsilon}^{n}\right) .
$$

That means

$$
J_{\varepsilon}\left(k_{\varepsilon}, q_{\varepsilon}, \pi_{\varepsilon}\right) \leq \liminf _{n \rightarrow+\infty} J_{\varepsilon}\left(k_{\varepsilon}^{n}, q_{\varepsilon}^{n}, \pi_{\varepsilon}^{n}\right)
$$

On the other hand, since $J_{\varepsilon}\left(k_{\varepsilon}^{n}, q_{\varepsilon}^{n}, \pi_{\varepsilon}^{n}\right) \leq d_{\varepsilon}+\frac{1}{n}$ and $\left.d_{\varepsilon} \leq J_{\varepsilon}\left(k_{\varepsilon}, q_{\varepsilon}, \pi_{\varepsilon}\right)\right)$ in particulary, we obey:

$$
\liminf _{n \rightarrow+\infty} J_{\varepsilon}\left(k_{\varepsilon}^{n}, q_{\varepsilon}^{n}, \pi_{\varepsilon}^{n}\right) \leq d_{\varepsilon} \leq J_{\varepsilon}\left(k_{\varepsilon}, q_{\varepsilon}, \pi_{\varepsilon}\right)
$$

It follows from (57) and (58)

$$
J_{\varepsilon}\left(k_{\varepsilon}, q_{\varepsilon}, \pi_{\varepsilon}\right)=d_{\varepsilon}=\inf _{(k, q, \pi) \in \mathcal{U}} J_{\varepsilon}(k, q, \pi) .
$$

The proof of Proposition 2.1 is achieved.

Proposition 2.2. Assume $h \in\left(L^{2}(Q)\right)^{N}$ such that $h \Theta$ belongs to $\left(L^{2}(Q)\right)^{N}$. Then, there exists $(\hat{\rho}, 0) \in V_{0}$ such that, the system $(\hat{k}, \hat{q}, \hat{\rho})$ is solution of the optimality systems

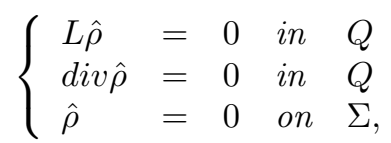

$$
\begin{aligned}
& \left\{\begin{array}{llll}
L^{*} \hat{q} & =h+\hat{k} \chi_{\omega_{T}} & & \text { in } \\
\operatorname{div} \hat{q} & =0 & & \text { in } \Sigma \\
\hat{q} & =0 & & \text { on } \Sigma \\
\hat{q}(T) & =0 & & \text { in } \Omega \\
\hat{q}(0) & =0 & & \text { in } \Omega
\end{array}\right.
\end{aligned}
$$

with

$$
\hat{k}=P \hat{\rho} \chi_{\omega_{T}}-\hat{\rho} \chi_{\omega_{T}} \in \mathcal{K}^{\perp}
$$


Proof. Since $\left(k_{\Theta}, q_{\Theta}, \pi_{\Theta}\right) \in \mathcal{U}$, for any $\varepsilon>0$, we have in particular:

$$
J_{\varepsilon}\left(k_{\varepsilon}, q_{\varepsilon}, \pi_{\varepsilon}\right)=\inf _{(k, q, \pi) \in \mathcal{U}} J_{\varepsilon}(k, q, \pi) \leq J_{\varepsilon}\left(k_{\Theta}, q_{\Theta}, \pi_{\Theta}\right) .
$$

That means from Definition of $J_{\varepsilon}$

$$
\forall \varepsilon>0, \frac{1}{2}\left\|k_{\varepsilon}\right\|_{L^{2}\left(\omega_{T}\right)}^{2}+\frac{1}{2 \varepsilon}\left\|L^{*} q_{\varepsilon}+\nabla \pi_{\varepsilon}-\left(h+k_{\varepsilon} \chi_{\omega_{T}}\right)\right\|_{L^{2}(Q)}^{2} \leq \frac{1}{2}\left\|k_{\Theta}\right\|_{L^{2}\left(\omega_{T}\right)}^{2} .
$$

So, we can conclude that $\left\|k_{\varepsilon}\right\|_{L^{2}\left(\omega_{T}\right)} \leq\left\|k_{\Theta}\right\|_{L^{2}\left(\omega_{T}\right)}=C$ and $\left\|L^{*} q_{\varepsilon}+\nabla \pi_{\varepsilon}-\left(h+k_{\varepsilon} \chi_{\omega_{T}}\right)\right\|_{L^{2}\left(\mathcal{O}_{T}\right)}^{2} \leq C \sqrt{\varepsilon}$.

Arguing as in Sections 2.1 and 2.1.2, we can show that there exists $(\tilde{q}, \tilde{\pi})$ such that

$$
L^{*} q_{\varepsilon}+\nabla \pi_{\varepsilon} \rightarrow L^{*} \tilde{q}+\nabla \tilde{\pi}, \quad \text { in }\left(\mathcal{D}^{\prime}(Q)\right)^{N} .
$$

\subsection{A characterization of the sequence control $k_{\varepsilon}$.}

$J_{\varepsilon}$ admits differentiability properties respect to the different variables $k, q$ and $\pi$. Consequently, since $J_{\varepsilon}\left(k_{\varepsilon}, q_{\varepsilon}, \pi_{\varepsilon}\right)=\inf _{(k, q, \pi) \in \mathcal{U}} J_{\varepsilon}(k, q, \pi)$ Euler-Lagrange Conditions at the first order can be formulated as

$$
\frac{J_{\varepsilon}\left(k_{\varepsilon}+\gamma\left(\bar{k}-k_{\varepsilon}\right), q_{\varepsilon}, \pi_{\varepsilon}^{n}\right)-J_{\varepsilon}\left(k_{\varepsilon}, q_{\varepsilon}, \pi_{\varepsilon}^{n}\right)}{\gamma} \geq 0,
$$

for any $\bar{k} \in \mathcal{K}^{\perp}, q_{\varepsilon}$ and $\pi_{\varepsilon}$.

However, some calculations give

$$
\begin{aligned}
& \frac{J_{\varepsilon}\left(k_{\varepsilon}+\gamma\left(\bar{k}-k_{\varepsilon}\right), q_{\varepsilon}, \pi_{\varepsilon}^{n}\right)-J_{\varepsilon}\left(k_{\varepsilon}, q_{\varepsilon}, \pi_{\varepsilon}^{n}\right)}{\gamma} \\
= & \frac{1}{2 \varepsilon \gamma}\left\{\left\|L^{*} q_{\varepsilon}+\nabla \pi_{\varepsilon}-\left[h+k_{\varepsilon}+\gamma\left(\bar{k}-k_{\varepsilon}\right) \chi_{\omega_{T}}\right]\right\|_{L^{2}(Q)}^{2}\right. \\
& \left.-\left\|L^{*} q_{\varepsilon}+\nabla \pi_{\varepsilon}-\left(h+k_{\varepsilon} \chi_{\omega_{T}}\right)\right\|_{L^{2}(Q)}^{2}\right\}+\frac{1}{2 \gamma}\left[\left\|k_{\varepsilon}+\gamma\left(\bar{k}-k_{\varepsilon}\right)\right\|_{L^{2}\left(\omega_{T}\right)}^{2}-\left\|k_{\varepsilon}\right\|_{L^{2}\left(\omega_{T}\right)}^{2}\right] \\
= & \frac{\gamma}{2}\left\|\bar{k}-k_{\varepsilon}\right\|_{L^{2}\left(\omega_{T}\right)}^{2}+\frac{\gamma}{2 \varepsilon}\left\|\left(\bar{k}-k_{\varepsilon}\right) \chi_{\omega_{T}}\right\|_{L^{2}\left(\omega_{T}\right)}^{2} \\
& +\int_{\omega_{T}} k_{\varepsilon}\left(\bar{k}-k_{\varepsilon}\right) d x d t-\int_{Q} \frac{1}{\varepsilon}\left[L^{*} q_{\varepsilon}+\nabla \pi_{\varepsilon}-\left(h+k_{\varepsilon} \chi_{\omega_{T}}\right)\right]\left(\bar{k}-k_{\varepsilon}\right) \chi_{\omega_{T}} d x d t .
\end{aligned}
$$

Let us set $\rho_{\varepsilon}=-\frac{1}{\varepsilon}\left[L^{*} q_{\varepsilon}+\nabla \pi_{\varepsilon}-\left(h+k_{\varepsilon} \chi_{\omega_{T}}\right)\right]$. We pass to the limit on $\gamma$ to 0 and we get

$$
\int_{\omega_{T}} k_{\varepsilon}\left(\bar{k}-k_{\varepsilon}\right) d x d t+\int_{\omega_{T}} \rho_{\varepsilon}\left(\bar{k}-k_{\varepsilon}\right) \chi_{\omega_{T}} d x d t \geq 0, \quad \forall \bar{k} \in \mathcal{K}^{\perp}
$$

We change successively $\bar{k}$ as $\bar{k}+k_{\varepsilon}$ and $-\bar{k}+k_{\varepsilon}$, we obtain

$$
\int_{\omega_{T}}\left(k_{\varepsilon}+\rho_{\varepsilon} \chi_{\omega_{T}}\right) \bar{k} d x d t=0, \quad \forall \bar{k} \in \mathcal{K}^{\perp}
$$

That means $k_{\varepsilon}+\rho_{\varepsilon} \chi_{\omega_{T}} \in \mathcal{K}$. Then since $k_{\varepsilon} \in \mathcal{K}^{\perp}$, we have the following characterization:

$$
k_{\varepsilon}=P \rho_{\varepsilon} \chi_{\omega_{T}}-\rho_{\varepsilon} \chi_{\omega_{T}},
$$

where $P$ designates the projector operator on $\mathcal{K}$. 
2.3.1. Some properties of $\rho_{\varepsilon}$ and some convergence results

Now, we fix here $k_{\varepsilon}$ and $\pi_{\varepsilon}$. Let $\bar{q} \in \mathcal{Q}$, Euler-Lagrange Conditions give

$$
\frac{J_{\varepsilon}\left(k_{\varepsilon}, q_{\varepsilon}+\gamma\left(\bar{q}-q_{\varepsilon}\right), \pi_{\varepsilon}^{n}\right)-J_{\varepsilon}\left(k_{\varepsilon}, q_{\varepsilon}, \pi_{\varepsilon}^{n}\right)}{\gamma} \geq 0, \quad \forall \bar{q} \in \mathcal{Q} .
$$

More precisely,

$$
\begin{aligned}
& \frac{J_{\varepsilon}\left(k_{\varepsilon}, q_{\varepsilon}+\gamma\left(\bar{q}-q_{\varepsilon}\right), \pi_{\varepsilon}^{n}\right)-J_{\varepsilon}\left(k_{\varepsilon}, q_{\varepsilon}, \pi_{\varepsilon}^{n}\right)}{\gamma} \\
= & \frac{1}{2 \varepsilon \gamma}\left[\left\|L^{*}\left(q_{\varepsilon}+\gamma\left(\bar{q}-q_{\varepsilon}\right)\right)+\nabla \pi_{\varepsilon}-\left(h+k_{\varepsilon}\right) \chi_{\omega_{T}}\right\|_{L^{2}(Q)}^{2}\right. \\
& \left.-\left\|L^{*} q_{\varepsilon}+\nabla \pi_{\varepsilon}-\left(h+k_{\varepsilon} \chi_{\omega_{T}}\right)\right\|_{L^{2}(Q)}^{2}\right] \\
= & \frac{\gamma}{2 \varepsilon}\left\|L^{*}\left(\bar{q}-q_{\varepsilon}\right)\right\|_{L^{2}(Q)}^{2}+\frac{1}{2 \varepsilon} \int_{Q} \rho_{\varepsilon} L^{*}\left(\bar{q}-q_{\varepsilon}\right) d x d t \geq 0 .
\end{aligned}
$$

We change successively $\bar{q}$ as $\bar{q}-q_{\varepsilon}$ and $-\bar{q}-q_{\varepsilon}$. After passing to the limit on $\gamma$, we get

$$
\int_{Q} \rho_{\varepsilon} L^{*} \bar{q} d x d t=0, \forall \bar{q} \in \mathcal{Q}
$$

So, after integrating by part over $Q$, we have for any $\bar{q} \in \mathcal{Q}$

$$
\int_{Q} L \rho_{\varepsilon} \bar{q} d x d t=\int_{Q} \rho_{\varepsilon} L^{*} \bar{q} d x d t+\left(\rho_{\varepsilon},-\frac{\partial \bar{q}}{\partial \nu}\right)_{L^{2}(\Sigma)}, \forall \bar{q} \in \mathcal{Q}
$$

On one hand, taking $\bar{q} \in \mathcal{Q}$ such that $\frac{\partial \bar{q}}{\partial \nu}=0$, we deduce

$$
L \rho_{\varepsilon}=0 \text { in } Q
$$

On the other hand, (65) implies $\left(\rho_{\varepsilon},-\frac{\partial \bar{q}}{\partial \nu}\right)_{L^{2}(\Sigma)}=0$ in $Q$ for any $\bar{q} \in \mathcal{Q}$, such that $L^{*} \bar{q}=0$ in $Q$. In this way, we have

$$
\rho_{\varepsilon}=0 \text { on } \Sigma \text {. }
$$

Moreover, we fix $k_{\varepsilon}, q_{\varepsilon}$. Employing again Euler-Lagrange Conditions, we have

$$
\frac{J_{\varepsilon}\left(k_{\varepsilon}, q_{\varepsilon}, \pi_{\varepsilon}^{n}+\gamma\left(\bar{\pi}-\pi_{\varepsilon}\right)\right)-J_{\varepsilon}\left(k_{\varepsilon}, q_{\varepsilon}, \pi_{\varepsilon}^{n}\right)}{\gamma} \geq 0, \quad \forall \bar{\pi} \in \mathcal{P}
$$

That means

$$
\frac{\gamma}{2 \varepsilon}\left\|\nabla\left(\bar{\pi}-\pi_{\varepsilon}\right)\right\|_{L^{2}(Q)}^{2}+\left(\nabla\left(\bar{\pi}-\pi_{\varepsilon}\right)\right)_{L^{2}(Q)} \geq 0
$$

We pass to the limit on $\gamma$, and we obtain

$$
\left(\nabla\left(\bar{\pi}-\pi_{\varepsilon}\right), \rho_{\varepsilon}\right)_{L^{2}(Q)} \geq 0
$$

We change successively $\bar{\pi}$ by $\bar{\pi}+\pi_{\varepsilon}$ and $-\bar{\pi}+\pi_{\varepsilon}$,

$$
\int_{Q} \rho_{\varepsilon} \nabla \bar{\pi} d x d t=0, \quad \forall \bar{\pi} \in \mathcal{P} .
$$


However, $\int_{Q} \rho_{\varepsilon} \nabla \bar{\pi} d x d t=\int_{\Sigma} \rho_{\varepsilon} \bar{\pi} d \sigma d t-\int_{Q} \bar{\pi} d i v \rho_{\varepsilon} d x d t$. So, thanks to $\rho_{\varepsilon}=0$ on $\Sigma$, (67) becomes

$$
\int_{Q} \bar{\pi} d i v \rho_{\varepsilon} d x d t=0, \quad \forall \bar{\pi} \in \mathcal{P} .
$$

We conclude that

$$
\operatorname{div} \rho_{\varepsilon}=0 \text { on } \Sigma .
$$

Consequently, from (65), (66) and (68), we get

$$
\left\{\begin{array}{rlll}
L \rho_{\varepsilon} & =0 & \text { in } & Q \\
\operatorname{div} \rho_{\varepsilon} & =0 & \text { in } & Q \\
\rho_{\varepsilon} & =0 & \text { on } & \Sigma
\end{array}\right.
$$

That means, $\left(\rho_{\varepsilon}, 0\right)$ is in $V_{0}$.

\subsubsection{An a priori bound of $\left\|\rho_{\varepsilon}\right\|_{L^{2}\left(\omega_{T}\right)}$}

From definition of norm \|\|$_{\Theta, 0}$, because $L \rho_{\varepsilon}=0$ in $Q$, then we have

$$
\left\|\left(\rho_{\varepsilon}, 0\right)\right\|_{\Theta, 0}^{2}=\int_{\omega_{T}}\left|\rho_{\varepsilon} \chi_{\omega_{T}}-P \rho_{\varepsilon} \chi_{\omega_{T}}\right|^{2} d x d t
$$

Moreover, in the previous section, we have observed that $k_{\varepsilon}=P \rho_{\varepsilon} \chi_{\omega_{T}}-\rho_{\varepsilon} \chi_{\omega_{T}}$, so $\left\|\left(\rho_{\varepsilon}, 0\right)\right\|_{\Theta, 0}=\left\|k_{\varepsilon}\right\|_{L^{2}\left(\omega_{T}\right)}$. $k_{\varepsilon}$ being bounded in $\left(L^{2}\left(\omega_{T}\right)\right)^{N}$ and $\left\{\left(\rho_{\varepsilon}, 0\right)\right\}_{\varepsilon>0}$ is also bounded in $V_{0}$. Following Remark 1.5, $\left(V_{0},\|\|_{\Theta, 0}\right)$ is an Hilbert space, consequently, there exists $(\tilde{\rho}, 0) \in V_{0}$ such that $\left(\rho_{\varepsilon}, 0\right)$ converges weakly to $(\tilde{\rho}, 0)$ in $V_{0}$. So, $\left(\rho_{\varepsilon}-\tilde{\rho}, 0\right)$ converges weakly to $(0,0)$ in $V_{0}$. Since, $\left(\rho_{\varepsilon}-\tilde{\rho}, 0\right) \in V_{0}$, thanks Lemma 1.2 , we can write

$$
\begin{aligned}
\int_{Q} \frac{1}{\Theta}\left|\rho_{\varepsilon}-\tilde{\rho}\right|^{2} d x d t \leq \quad & C\left[\int_{Q}\left|L\left(\rho_{\varepsilon}-\tilde{\rho}\right)\right|^{2} d x d t+\right. \\
& \left.\int_{\omega_{T}}\left|\left(\rho_{\varepsilon}-\tilde{\rho}\right) \chi_{\omega_{T}}-P\left(\rho_{\varepsilon}-\tilde{\rho}\right) \chi_{\omega_{T}}\right|^{2} d x d t\right] .
\end{aligned}
$$

The right hand is bounded independently on $\varepsilon$ because $L \rho_{\varepsilon}=0$ in $Q$ and $k_{\varepsilon}=\rho_{\varepsilon} \chi_{\omega_{T}}-P \rho_{\varepsilon} \chi_{\omega_{T}}$ is bounded. It results that $\frac{1}{\Theta}\left(\rho_{\varepsilon}-\tilde{\rho}\right)$ is bounded in $\left(L^{2}(Q)\right)^{N}$.

Furthermore, since

$$
\begin{aligned}
\int_{\omega_{T}} \frac{1}{\Theta}\left|P\left(\rho_{\varepsilon}-\tilde{\rho}\right) \chi_{\omega_{T}}\right|^{2} d x d t \leq 2 & {\left[\int_{\omega_{T}} \frac{1}{\Theta}\left|\left(\rho_{\varepsilon}-\tilde{\rho}\right)^{2} \chi_{\omega_{T}}\right|^{2} d x d t+\right.} \\
& \left.\int_{\omega_{T}} \bar{\Theta}\left|\left(\rho_{\varepsilon}-\tilde{\rho}\right) \chi_{\omega_{T}}-P\left(\rho_{\varepsilon}-\tilde{\rho}\right) \chi_{\omega_{T}}\right|^{2} d x d t\right],
\end{aligned}
$$

we deduce that $\frac{1}{\Theta} P\left(\rho_{\varepsilon}-\tilde{\rho}\right) \chi_{\omega_{T}}$ is bounded in $L^{2}\left(\omega_{T}\right)$ and then from equivalence of norm, $P\left(\rho_{\varepsilon}-\tilde{\rho}\right) \chi_{\omega_{T}}$ is also bounded in $\left(L^{2}\left(\omega_{T}\right)\right)^{N}$. Let $\alpha$ assigned in $\left(L^{2}\left(\omega_{T}\right)\right)^{N}$ such that $P\left(\rho_{\varepsilon}-\tilde{\rho}\right) \chi_{\omega_{T}} \rightarrow \alpha$ in $\left(L^{2}\left(\omega_{T}\right)\right)^{N}$.

Moreover, using Phytagore's Theorem, we have

$$
\left\|\left(\rho_{\varepsilon}-\tilde{\rho}\right) \chi_{\omega_{T}}\right\|_{L^{2}\left(\omega_{T}\right)}^{2}=\left\|P\left(\rho_{\varepsilon}-\tilde{\rho}\right) \chi_{\omega_{T}}\right\|_{L^{2}\left(\omega_{T}\right)}^{2}+\left\|\left(\rho_{\varepsilon}-\tilde{\rho}\right) \chi_{\omega_{T}}-P\left(\rho_{\varepsilon}-\tilde{\rho}\right) \chi_{\omega_{T}}\right\|_{L^{2}\left(\omega_{T}\right)}^{2},
$$


and we deduce that $\left(\rho_{\varepsilon}-\tilde{\rho}\right) \chi_{\omega_{T}}$ is bounded in $\left(L^{2}\left(\omega_{T}\right)\right)^{N}$. Extracting a subsequence, we can assume that the sequence $\left(\rho_{\varepsilon}-\tilde{\rho}\right) \chi_{\omega_{T}}$ converges weakly in $\left(L^{2}\left(\omega_{T}\right)\right)^{N}$. Let us set $\beta \in\left(L^{2}\left(\omega_{T}\right)\right)^{N}$ such that $\left(\rho_{\varepsilon}-\tilde{\rho}\right) \chi_{\omega_{T}} \rightarrow \beta$. In particular, since $P$ is a continuous operator, we also have $P\left(\rho_{\varepsilon}-\tilde{\rho}\right) \chi_{\omega_{T}} \rightarrow P \beta$ in $\left(L^{2}\left(\omega_{T}\right)\right)^{N}$.

It results that

$$
\alpha=P \beta
$$

and

$$
\left(\rho_{\varepsilon}-\tilde{\rho}\right) \chi_{\omega_{T}}-P\left(\rho_{\varepsilon}-\tilde{\rho}\right) \chi_{\omega_{T}} \rightarrow \beta-P \beta .
$$

We show that $\beta=0$. Indeed, according to definition of the bilinear form $a_{\Theta, 0}$, since $\left(\rho_{\varepsilon}-\tilde{\rho}, 0\right)$ converges weakly to $(0,0)$ in $V_{0}$, for any $(\phi, 0)$ in $V_{0}$,

$$
\int_{Q} L\left(\rho_{\varepsilon}-\tilde{\rho}\right) L \phi d x d t+\int_{\omega_{T}}\left[\left(\rho_{\varepsilon}-\tilde{\rho}\right) \chi_{\omega_{T}}-P\left(\rho_{\varepsilon}-\tilde{\rho}\right) \chi_{\omega_{T}}\right]\left(\phi \chi_{\omega_{T}}-P \phi \chi_{\omega_{T}}\right) d x d t
$$

tends to 0 when $\varepsilon$ tends to 0 .

Taking $(\phi, 0)$ in $V_{0}$ such that $L \phi=0$ in $Q,(71)$ becomes successively

$$
\int_{\omega_{T}}\left[\left(\rho_{\varepsilon}-\tilde{\rho}\right) \chi_{\omega_{T}}-P\left(\rho_{\varepsilon}-\tilde{\rho}\right) \chi_{\omega_{T}}\right]\left(\phi \chi_{\omega_{T}}-P \phi \chi_{\omega_{T}}\right) d x d t=0
$$

tends to 0 as $\varepsilon$. That means, because

$$
\int_{\omega_{T}}\left[\left(\rho_{\varepsilon}-\tilde{\rho}\right) \chi_{\omega_{T}}-P\left(\rho_{\varepsilon}-\tilde{\rho}\right) \chi_{\omega_{T}}\right]\left(\phi \chi_{\omega_{T}}-P \phi \chi_{\omega_{T}}\right) d x d t=\int_{\omega_{T}}\left[\left(\rho_{\varepsilon}-\tilde{\rho}\right) \chi_{\omega_{T}}-P\left(\rho_{\varepsilon}-\tilde{\rho}\right) \chi_{\omega_{T}}\right] \phi \chi_{\omega_{T}} d x d t
$$

For any $(\phi, 0)$ in $V_{0}$ such that $L \phi=0$ in $Q$,

$$
\int_{\omega_{T}}\left[\left(\rho_{\varepsilon}-\tilde{\rho}\right) \chi_{\omega_{T}}-P\left(\rho_{\varepsilon}-\tilde{\rho}\right) \chi_{\omega_{T}}\right] \phi \chi_{\omega_{T}} d x d t
$$

tends to 0 as $\varepsilon$.

From (70), it comes $\int_{\omega_{T}}(\beta-P \beta) \phi d x d t=0$. Consequently, we get, $\beta-P \beta=0$. In other words, $\beta$ is in $\mathcal{K}$.

Now, because $\beta-P \beta=0,(71)$ is reduced to

$$
\int_{Q} L\left(\rho_{\varepsilon}-\tilde{\rho}\right) L \phi d x d t
$$

tends to 0 when $\varepsilon$ tends to 0 , for any $(\phi, 0)$ in $V_{0}$.

Also, $L \rho_{\varepsilon}=0$ is in $Q$ and so we have $L\left(\rho_{\varepsilon}-\tilde{\rho}\right)$ converges strongly to $L \tilde{\rho}$ in $\left(L^{2}(Q)\right)^{N}$. Then, taking $\phi=\tilde{\rho}$ in (72), we obtain $\int_{Q}|L \tilde{\rho}|^{2} d x d t=0$. We deduce $L \tilde{\rho}=0$ in $Q$ and so successively, $L \tilde{\rho} \chi_{\omega_{T}}=0$ and $L\left(\rho_{\varepsilon}-\tilde{\rho}\right) \chi_{\omega_{T}} \rightarrow 0$. By uniqueness, we get $L \beta=0$ in $\omega_{T}$.

Finally, $\beta$ is such that

$$
\left\{\begin{aligned}
L \beta & =0 \\
\beta & \in \mathcal{K} .
\end{aligned} \text { in } \omega_{T}\right.
$$

Applying hypothesis (21), we get $\beta=0$.

Consequently, we have $k_{\varepsilon}=P \rho_{\varepsilon} \chi_{\omega_{T}}-\rho_{\varepsilon} \chi_{\omega_{T}} \rightarrow P \tilde{\rho} \chi_{\omega_{T}}-\tilde{\rho} \chi_{\omega_{T}}$ and $\tilde{\rho}$ is such that $L \tilde{\rho}=0$ in $Q$. 


\subsection{New definition of $\hat{k}$}

We go back to the definition given to $J_{\varepsilon}$, and by particulary from (59), we have

$$
J_{\varepsilon}\left(k_{\varepsilon}, q_{\varepsilon}, \pi_{\varepsilon}\right) \leq J_{\varepsilon}(k, q, \pi), \quad \forall(k, q, \pi) \in \mathcal{U} .
$$

The notations used here are as in the end of Section 1.2. We recall that $\frac{1}{2}\|\hat{k}\|_{L^{2}\left(\omega_{T}\right)}^{2}=\min _{k \in \mathcal{E}} \frac{1}{2}\|k\|_{L^{2}\left(\omega_{T}\right)}^{2}$. Since $\hat{k} \in \mathcal{E}$, there exists $(\hat{q}, \hat{\pi})$ such that $L^{*} \hat{q}+\nabla \hat{\pi}-(h+\hat{k}) \chi_{\omega_{T}}=0$ in $Q$, $\operatorname{div} \hat{q}=0, \hat{q}=0$ on $\Sigma, \hat{q}(T)=\hat{q}(0)=0$ in $\Omega$.

We put $k=\hat{k}, q=\hat{q}$ and $\hat{\pi}$ in (73), then we have more simply

$$
\frac{1}{2}\left\|k_{\varepsilon}\right\|_{L^{2}\left(\omega_{T}\right)}^{2} \leq \frac{1}{2}\|\hat{k}\|_{L^{2}\left(\omega_{T}\right)}^{2} .
$$

After passing to low limit on $\varepsilon$, we obtain

$$
\|\tilde{k}\|_{L^{2}\left(\omega_{T}\right)} \leq \liminf _{\varepsilon \rightarrow 0}\left\|k_{\varepsilon}\right\|_{L^{2}\left(\omega_{T}\right)} \leq\|\hat{k}\|_{L^{2}\left(\omega_{T}\right)}
$$

From (63), since $k_{\varepsilon}$ tends to $\tilde{k}$. So, we have $\left\|L^{*} \tilde{q}+\nabla \tilde{\pi}-\left(h+\tilde{k} \chi_{\omega_{T}}\right)\right\|_{L^{2}(Q)}=0$. That is $\tilde{k} \in \mathcal{E}$. Since the solution of the problem $\min _{k \in \mathcal{E}} \frac{1}{2}\|k\|_{L^{2}\left(\omega_{T}\right)}^{2}$, is unique, we conclude that $\tilde{k}=\hat{k}$.

We have the following characterization $\hat{k}=\tilde{\rho}-P \tilde{\rho}$ and the statement of Proposition 2.2 occurs by taking $\hat{\rho} \chi_{\omega_{T}}=\tilde{\rho}$.

\section{Proof of Theorem 0.8. A Discriminating Sentinel}

This section is devoted to apply the results obtained in the Section 3. We go back to Section 1. In particular, we have observed that the determination of a Sentinel is completely in solving problem 16 . However, (16) coincides to the problem (15) when $h=h_{0} \chi_{\mathcal{O}}+k_{0} \chi_{\omega_{T}}$. More precisely, by taking $\hat{\rho}$ such that $\left\{\begin{aligned} L \hat{\rho} \chi_{\omega_{T}} & =0 \\ \hat{\rho} \chi_{\omega_{T}} & \in \mathcal{K}\end{aligned}\right.$ in $\quad \omega_{T} \quad$ and according to Theorem 0.6 there exists a system $\{\hat{k}, \hat{q}, \hat{\pi}\}$ defined by $\hat{k}=\hat{\rho}-P \hat{\rho}$, $\hat{q}=q(\hat{k})$ solves $(16)$.

By taking $\hat{w}=k_{0}+\hat{k}$, Definition 0.1 follows and it becomes

Definition 3.1. The function $\hat{\mathcal{S}}$ defined as

$$
\hat{\mathcal{S}}(\lambda, \tau)=\int_{0}^{T} \int_{\mathcal{O}} h_{0} y(x, t ; \lambda, \tau) d x d t+\int_{0}^{T} \int_{\omega} \hat{w} y(x, t ; \lambda, \tau) d x d t
$$

is a discriminating Sentinel.

Taking $\mathcal{O}=\omega$ and $k_{0}=-P h_{0}$, we observe that Definition 3.1 is an extension of the work due to J.L. Lions in [15]. In this case, $\hat{\mathcal{S}}$ becomes $\hat{\mathcal{S}}(\lambda, \tau)=\int_{0}^{T} \int_{\mathcal{O}}\left(h_{0}+\hat{\rho}+P\left(h_{0}+\hat{\rho}\right)\right) y(x, t ; \lambda, \tau) d x d t$ and coincides with the well known version of Sentinel presented in [15].

Moreover, the insensitivity condition (5) or more precisely (10) becomes

$$
\int_{0}^{T} \int_{\mathcal{O}} h_{0} y_{\tau}(x, t) d x d t+\int_{0}^{T} \int_{\omega} \hat{w} y_{\tau}(x, t) d x d t=0,
$$

where $y_{\tau}(x, t)$ is defined by $(9)$. 


\subsection{A use of the concept of sentinel: The identification of the unknown distributed pollution term}

Let us now, present a use of the concept of sentinel applied to some perturbed Navier-Stokes system.

The notations used here are again those of the previous sections. Let $\hat{\mathcal{S}}_{\mid \text {obs }}$ be the global information provided by the observation $y_{\mid o b s}$. Since $\hat{w}$ is insensitive with respect to the interfering system $\left\{m_{i}\right\}_{i=1, \cdots, M}$, from Definition 3.1 we have

$$
\begin{aligned}
\hat{\mathcal{S}}_{\mid o b s}(\lambda, \tau)-\hat{\mathcal{S}}(0,0) & =\int_{0}^{T} \int_{\Omega}\left(h_{0} \chi_{\mathcal{O}}+\hat{w} \chi_{\omega}\right)\left(y_{\mid o b s}-y_{0}\right) d x d t \\
& =\int_{0}^{T} \int_{\Omega}\left(h_{0} \chi_{\mathcal{O}}+\hat{w} \chi_{\omega}\right)\left(m_{0}-y_{0}\right) d x d t
\end{aligned}
$$

However, since we have $\hat{\mathcal{S}}_{\mid \text {obs }}(\lambda, \tau)-\hat{\mathcal{S}}(0,0)=\lambda \frac{\partial \hat{\mathcal{S}}}{\partial \lambda}(0,0)+0(\lambda, \tau)$ and $\frac{\partial \hat{\mathcal{S}}}{\partial \lambda}(0,0)=\int_{0}^{T} \int_{\Omega}\left(h_{0} \chi_{\mathcal{O}}+\hat{w} \chi_{\omega}\right) y_{\lambda} d x d t$ we get

$$
\lambda \int_{0}^{T} \int_{\Omega}\left(h_{0} \chi_{\mathcal{O}}+\hat{w} \chi_{\omega}\right) y_{\lambda} d x d t=\int_{0}^{T} \int_{\Omega}\left(h_{0} \chi_{\mathcal{O}}+\hat{w} \chi_{\omega}\right)\left(m_{0}-y_{0}\right) d x d t
$$

From (2), $y_{\lambda}$ is the unique solution of the linear Navier-Stokes system

$$
\left\{\begin{array}{rllll}
L y_{\lambda}+\nabla p_{\lambda} & = & \hat{\xi} & \text { in } & Q \\
d i v y_{\lambda} & = & 0 & \text { in } & Q \\
y_{\lambda} & = & 0 & \text { on } & \Sigma \\
y_{\lambda}(0) & = & 0 & \text { on } & \Omega .
\end{array}\right.
$$

Now, we designate as $\hat{q}\left(h_{0}\right)$ the unique solution of (11) depending on $h_{0}$.

Multiplying (11) by $y_{\lambda}$, we obtain after integrating by part over $Q$,

$$
\int_{0}^{T} \int_{\Omega}\left(h_{0} \chi_{\mathcal{O}}+\hat{w} \chi_{\omega}\right) y_{\lambda} d x d t=\int_{0}^{T} \int_{\Omega} \hat{q}\left(h_{0}\right) L y_{\lambda} d x d t
$$

Since $\operatorname{div} \hat{q}\left(h_{0}\right)=0$ in $Q$, we also have

$$
\begin{aligned}
\int_{0}^{T} \int_{\Omega}\left(h_{0} \chi_{\mathcal{O}}+\hat{w} \chi_{\omega}\right) y_{\lambda} d x d t & =\int_{0}^{T} \int_{\Omega} \hat{q}\left(h_{0}\right)\left(L y_{\lambda}+\nabla p_{\lambda}\right) d x d t \\
& =\int_{0}^{T} \int_{\Omega} \hat{q}\left(h_{0}\right) \hat{\xi} d x d t
\end{aligned}
$$

It results that the unknown pollution term $\lambda \hat{\xi}$ can be defined as follows

$$
\begin{aligned}
\int_{0}^{T} \int_{\Omega} \hat{q}\left(h_{0}\right)\{\lambda \hat{\xi}\} d x d t & =\hat{\mathcal{S}}_{\mid o b s}(\lambda, \tau)-\hat{\mathcal{S}}(0,0) \\
& =\int_{0}^{T} \int_{\Omega}\left(h_{0} \chi_{\mathcal{O}}+\hat{w} \chi_{\omega}\right)\left(m_{0}-y_{0}\right) d x d t
\end{aligned}
$$

Thus, the proof of the second part of Theorem 0.8 is complete.

\section{REFERENCES}

[1] O. Bordat, C. Fabre, "Controls insentisitizing the norm of the solution of a semililear heat equation" J. of Mathem. Analysis and Applications 195 (1995) pp. 658-683. 
[2] O. Bordat, M. Gonnzalez-Burgos, R. Perz-Garcia "Insentisitizing controls for a semililear heat equation with a superlinear nonlinearity" C.R.A.S Paris, Ser. I. 335 (2002) pp. 677-682.

[3] M. Boulakia "Existence of weak solutions for the motion of an elastic structure in a incompressible viscous fluid." C.R.A.S Paris, Ser. I. 336, (12) (2003) pp. 985-990.

[4] M. Boulakia "Existence of weak solutions for an interaction problem between an elastic structure and a compressible viscous fluid." C.R.A.S Paris, Ser. I. 340, (2) (2005) pp. 113-118.

[5] J.-M. Coron "On the controllability od the 2-D incompressible Navier-Stokes equations with the Navier slip boundary conditions". ESAIM:COCV, 1 (1996) pp. 35-75.

[6] J.-M. Coron, A. Fursikov "Global exact controllability of the 2D Navier-Stokes equations on a manifold without boundary". Russian J. Math. Phys., 4 (1996) pp. 1-19.

[7] D. Coutand, S. Shkoller "The interaction between elastodynamics and the Navier-Stokes equations". Arch. Rat. Mech. Anal, (2005) to appear.

[8] E. Fernandez-Cara, G. Garcia, A. Osses, "Inégalités et contrôle insensibilisant d'un modèle océanographique quasigéostrophique", Congrès d'analyse numérique, Canum. (2003).

[9] E. Fernandez-Cara, S. Guerrero, O.Yu. Imanuvilov, J.P. Puel, "Local exact controllability of the Navier-Stokes Syste"m, J. Math. Pures Appl. 83 (2004) pp. 1501-1542.

[10] A. Fursikov "Exact boundary zero controllability of three dimensional Navier-Stokes equations". Journal of Dynamical and control system, 1 (1995) pp. 325-350.

[11] A. Fursikov, O. Yu. Imanuvilov "On exact boundary zero-controllability of two-dimensional Navier-Stokes equations". Acta Applicandae Mathematice, 37 (1994) pp. 67-76.

[12] A. Fursikov, O. Yu. Imanuvilov "Exact controllability of the Navier-Stokes and Boussinesq equations". Russ. Math. Surveys, 54 (3), (1999) pp. 565-618.

[13] L. De Teresa, "Insentisitizing controls for a semililear heat equation. Commun. in Partial Differential Equations", 25, (2000), pp 39-72.

[14] J.L. Lions, "Exact controllability, stabilization and perturbations for distributed systems, J. Von Neumann Lecture, Boston 1986,", SIAM review, 30,, (1988), pp. 1-68 .

[15] J.L. Lions, "Sentinelles pour les systèmes distribués a données incomplètes", Masson, Paris (1992).

[16] J.L. Lions, E. Magenes, "Problèmes aux limites non homogènes et applications" Dunod, Paris (1968).

[17] J.L. Lions, "Quelques notions dans l'Analyse et le Contrôle de systèmes à données incomplètes". Proceeding of the XIth Congress on Differential Equations and Applications/First Congress on Applied Mathematics, University of Malálaga, Malálaga, (1990) pp. 43-54.

[18] J.L. Lions, G. Prodi, "Sur les sentinelles des systèmes distribués". C.R.A.S. Paris, 307, Ser. I (1988), pp. 819-823

[19] J.L. Lions, E. Zuazua, "Exact boundary controllability of Galerking's approximations of Navier-Stokes equations". Annali Scuola Norm. Sup. Pisa, XXVI, (1988), pp. 605-621

[20] O. Nakoulima, "Contrôlabilité à zéro avec contraintes sur le contrôle". C.R.A.S. Paris, Ser. I 339 (2004), pp 405-410.

[21] O.A. Ladyzenskaya, "Solution globale du problème aux limites pour l'équation de Navier-Stokes en deux variables d'espaces", Doklay Akad. Nauk. SSSR, 123, (1958), pp. 427-429.

[22] R. Temam, "Navier-Equations. Theory and Numerical Analysis", Stud. Math. Appl., North-Holland, Amsterdam-New YorkOxford, (1977). 